\title{
Sampling and analysis of chemical element concentration distribution in rock units and orebodies
}

\author{
F. P. Agterberg \\ Geological Survey of Canada, 601 Booth Street, Ottawa, Ont. K1A 0E8, Canada \\ Correspondence to: F. P. Agterberg (agterber@nrcan.gc.ca)
}

Received: 19 March 2010 - Revised: 22 February 2011 - Accepted: 17 December 2011 - Published: 5 January 2012

\begin{abstract}
Existing sampling techniques applied within known orebodies, such as sampling along mining drifts, yield element concentration values for larger blocks of ore if they are extended into their surroundings. The resulting average concentration values have relatively small "extension variance". These techniques can be used for multifractal modeling as well as ore reserve estimation approaches. Geometric probability theory can aid in local spatial covariance modeling. It provides information about increase of variability of element concentration over short distances exceeding microscopic scale. In general, the local clustering of ore crystals results in small-scale variability known as the "nugget effect". Parameters to characterize spatial covariance estimated from ore samples subjected to chemical analysis for ore reserve estimation may not be valid at local scale because of the nugget effect. The novel method of local singularity mapping applied within orebodies provides new insights into the nature of the nugget effect. Within the Pulacayo orebody, Bolivia, local singularity for zinc is linearly related with logarithmically transformed concentration value. If there is a nugget effect, moving averages resulting from covariance models or estimated by other methods that have a smoothing effect, such as kriging, can be improved by incorporating local singularities indicating local element enrichment or depletion. Although there have been many successful applications of the multifractal binomial $/ p$ model, its application within the Pulacayo orebody results in inconsistencies, indicating some shortcomings of this relatively simple approach. Local singularity analysis and universal multifractal modeling are two promising new approaches to improve upon results obtained by commonly used geostatistical techniques and use of the binomial $/ p$ model. All methods in this paper are illustrated using a single example (118 Pulacayo zinc values), and several techniques are applied to other orebody
\end{abstract}

datasets (Whalesback copper deposit, Witwatersrand goldfields and Black Cargo titanium deposit). Additionally, it is discussed that nugget effects exist in a binary series of alternating mostly gneiss and metabasite previously derived from KTB borehole velocity and lithology logs, and within a series of 2796 copper concentration values from this same drillhole.

\section{Introduction}

Most geological maps display bedrock as a mosaic of distinct rock units of different composition and age. Small rock samples are taken and subjected to chemical analysis. Normally, the resulting chemical element concentration values are used to help with rock identification and to describe the physico-chemical processes that led to the patterns of rock units on the geological map and its three-dimensional extensions into depth. Although orebodies and hydrocarbon deposits generally occupy relatively small volumes within the Earth's crust, they are targets of intense exploration including chemical determinations both before and after discovery. These targets of economic interest often possess fractal characteristics that cannot be fully explained without the use of nonlinear concepts. This paper is concerned with spatial patterns of chemical element concentration distribution that are best described as multifractals, which are superimposed on the multifaceted mosaic of the geological map.

During the past $40 \mathrm{yr}$, the fractal geometry of many natural features in Nature has become widely recognized (see e.g. Mandelbrot, 1983; Barnsley, 1988; Turcotte, 1997). Fractals in geology either represent the end products of numerous, more or less independent processes (e.g. coastlines and topography), or they result from nonlinear processes, that took 
place long ago within the Earth's crust. Although a great variety of fractals can be generated by relatively simple algorithms, theory needed to explain fractals of the second kind generally is not so simple, because previously neglected nonlinear terms have to be inserted into existing linear, deterministic equations. Several types of patterns are best modeled as multifractals, which are spatially intertwined fractals (Stanley and Meakin, 1988). Most progress in multifractal theory development has been made in geophysics to study nonlinear processes including cloud formation and rainfall. Lovejoy and Schertzer (2007), Lovejoy et al. (2008) and Cheng (2008) show that scaling and multifractal fields also exist within solid Earth.

In this paper special attention will be paid to sampling problems which arise because chemical concentration values for small rock samples must be extrapolated over much larger rock masses in order to describe the multifractal fields. Such extrapolations remain subject to uncertainty. Use will be made of geostatistical theory originally developed by Matheron (1962), whose approach is also explained in various geostatistical textbooks including Journel and Huijbregts (1978) and Cressie (1991). Nearly all publications by Matheron including his manual on the theory of regional variables and its applications (Matheron, 1971) are now freely available on a website maintained by the Ecole Nationale Supérieure des Mines de Paris (http://cg.endmp. fr.bibliotheque/cgi-bin/public/bibli_index.cgi). Matheron (1962) initially based his geostatistical theory on the principle of "similitude" underlying the model of de Wijs (1951). Later, Mandelbrot (1983) recognized that it can be said that de Wijs (1951) developed the first multifractal now better known as the binomial/ $p$ model (see e.g. Lovejoy and Schertzer, 2007). Krige (1978) demonstrated that the model of de Wijs could be applied to hundreds of thousands of gold assays, at scales ranging from local sampling scale; from Witwatersrand goldfields (see also Mandelbrot, 1995).

To illustrate application of his model, De Wijs (1951) used a series of 118 zinc concentration values from samples taken at a regular $(2 \mathrm{~m})$ interval along a horizontal drift in the $\mathrm{Pu}-$ lacayo Mine, Bolivia (Fig. 1). This series was used extensively for later study, as well by Matheron (1964), and several other authors including, most recently, Chen et al. (2007) and Lovejoy and Schertzer (2007). This example will again be used in this paper. Geological background on the Pulacayo orebody will be provided and consideration paid to the question of how representative is this example of ore deposits in general. As explained in more detail elsewhere (e.g. Agterberg, 2007a, b), de Wijs assumed that, if a block of ore is divided into halves, the ratio of average element concentration values for the halves is equal to the same constant regardless of the size of the block that is divided into halves. If greater value is divided by lesser value, this ratio can be written as $\eta>1$. Matheron (1962) generalized the original model of de Wijs by introducing the concept of "absolute dispersion" written as $\alpha=(\ln \eta)^{2} / \ln 16$. This approach is equivalent to

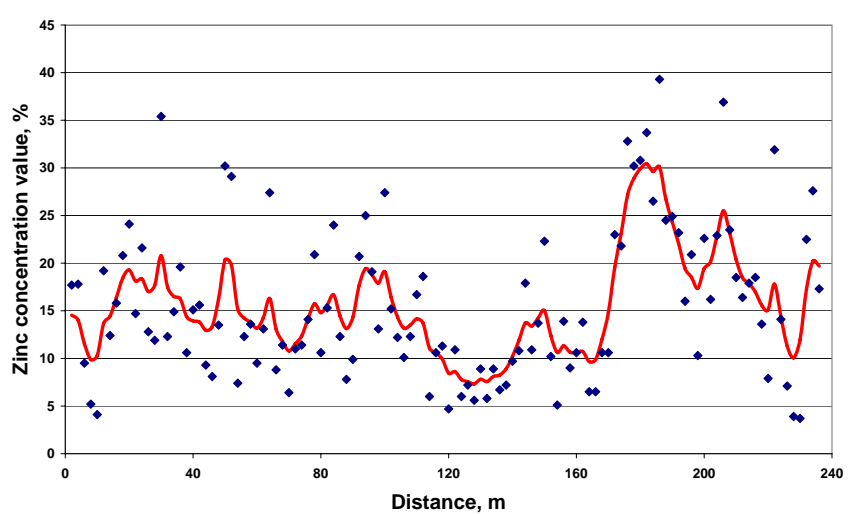

Fig. 1. Pulacayo Mine zinc concentration values for 118 channel samples along horizontal drift. Sampling interval is $2 \mathrm{~m}$. Original data (blue diamonds) are from de Wijs (1951) and "signal" (red line) retained after removal of "nugget effect" is from Agterberg (1974).

what is now better known as scale invariance. It leads to the more general equation $\sigma^{2}(\ln x)=\alpha \times \ln V / v$ where $\sigma^{2}(\ln$ $x$ ) represents logarithmic variance of element concentration values $x$ in smaller blocks with volume $v$ contained within a larger block of ore with volume $V$.

Two geostatistical topics of practical interest are existence of "sill" and "nugget effect" (see e.g. Journel and Huijbregts, 1968 , or Cressie, 1991). Suppose $\gamma(h)$ represents the semivariogram, which is half the variance of the difference between values separated by lag distance $h$. Semivariogram values normally increase when $h$ is increased until a sill value is reached for large distances. If element concentration values are subject to second-order stationarity, $\gamma(h)=$ $\sigma^{2}\left(1-\rho_{h}\right)$ where $\sigma^{2}$ represents variance and $\rho_{h}$ is the autocorrelation function. The sill is reached when there is no spatial autocorrelation or $\gamma(h)=\sigma^{2}$. If regional trends can be separately fitted to element concentration values, the residuals from the resulting regional, systematic variation may become second-order stationary because the overall mean in the study area then is artificially set equal to zero (see Sects. 4.3 and 4.4 for examples of this approach). Within most rock types such as granite or sandstone, randomness of chemical concentration is largely restricted to microscopic scale and sills for compositional data are reached over very short distances. The nugget effect occurs when extrapolation of $\gamma(h)$ towards the origin $(h \rightarrow 0)$ from observed element concentration values yields estimates with $\gamma(h)>0$ (or $\rho_{h}<1$ ). Often the nugget effect arises when there is strong local autocorrelation that cannot be detected because locations of samples subjected to chemical analysis are too far apart to describe it adequately.

If a segment of the Earth's crust is sampled and element concentration values are determined on the resulting rock samples, the spatial variability of the chemical determinations generally can be subdivided into a number of separate components. In some applications the original data are 
stochastic in that they can be described by random functions. However, often the main component of spatial variability is deterministic, either because it is related to differences between rock units separated by discontinuities (contacts), or because there are regional trends. The latter can be extracted from the data by a variety of methods; e.g. by trend surface analysis, calculation of moving averages with or without weights that are powers of the inverse of distance, by various methods of kriging, by using splines, or by means of other methods of signal extraction. After extraction of a deterministic component, the residuals generally are stochastic in that they can be described by means of spatial random functions. In the simplest case, these residuals are uncorrelated and their correlogram is a Dirac delta function representing white noise. Measurement errors would create white noise. If extrapolation towards the origin by means of a function fitted to the correlogram results in a variance that significantly exceeds variance due to measurement errors, this indicates existence of a nugget effect implying strong autocorrelation over short distances. In this paper, special attention will be paid to nugget effects, which probably are due to spatial clustering of ore crystals.

Matheron (1989) has pointed out that in rock sampling there are two possible infinities if number of samples is increased indefinitely: either the sampling interval is kept constant so that more rock is covered, or size of study area is kept constant whereas sampling interval is decreased. These two possible sampling schemes provide additional information on sample neighbourhood, for sill and nugget effect, respectively. In practice, the exact form of the nugget effect usually remains unknown because extensive sampling would be needed at a scale that exceeds microscopic scale but is less than scale of sampling space commonly used for ore deposits or other geological bodies. Nevertheless, there are now several methods by means of which the nugget effect can be studied. The de Wijs zinc data set is rather small (118 values). Because of this, larger data sets will be analyzed as well. As an example taken from another geoscience field, it will be discussed in the text that alternating, detrended lithologies over a length of about $7 \mathrm{~km}$ in the KTB borehole (Goff and Hollinger, 1999) show a small-scale nugget effect. Additionally, a series of 2796 copper concentration values for chip samples taken at 2-m intervals along the Main KTB borehole show a persistent nugget effect that will be analyzed separately. Cheng (1999, 2005, 2006) has proposed a new model for incorporating spatial association and singularity in interpolation of exploratory data. The first two sections in this paper will mainly deal with geostatistics and applications of geometrical probability theory to sampling. It will be shown later in the paper that Cheng's approach provides a novel way of incorporating the nugget effect. The iterative algorithm proposed by Chen et al. (2007) for local singularity mapping will be extended until full convergence is reached. Local singularities obtained during this process provide new information on the nature of the nugget effect.

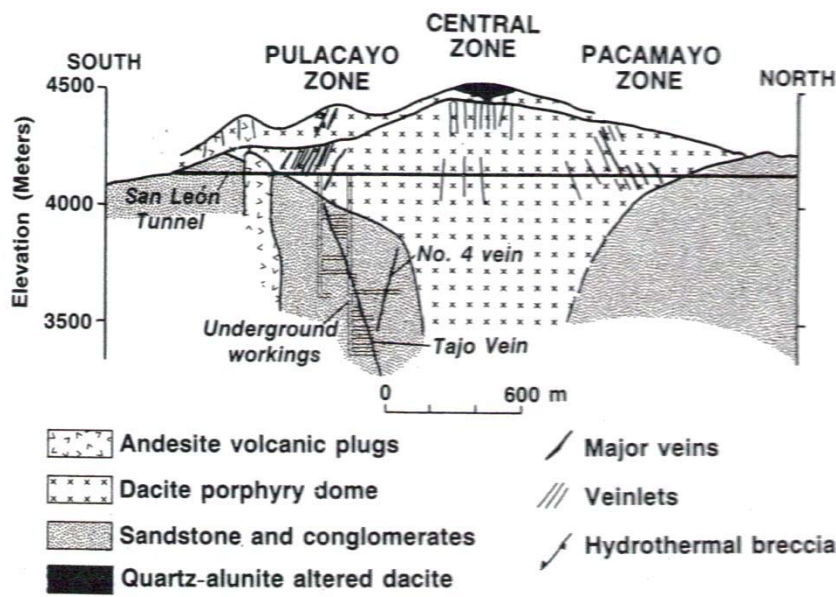

Fig. 2. Simplified cross section of Pulacayo dome with steeply dipping Tajo Vein (after Pinto-Vásquez, 1993). Mining level depths were measured downward from San León Tunnel.

At the end of the paper, spectral analysis of element concentration values will be briefly discussed in connection with probable existence of the nugget effect and in the context of universal multifractal modeling results obtained by Lovejoy and Schertzer (2007) for the Pulacayo Mine in Bolivia. The purpose of the analyses described in this paper is to help construct viable models that honor the observations and extrapolate from limited spatial sampling to smaller or larger volumes of rocks including orebodies, so that unbiased and relatively precise average element concentrations are obtained for these volumes, which can have different shapes. If possible, each estimated average concentration value for a volume of rock should be accompanied by realistic estimates of the uncertainties associated with it.

\section{Basic statistical analyses of mining assays}

\subsection{Geological setting of Pulacayo Mine}

The geological setting of the Pulacayo Mine and genesis of the sphalerite-quartz ore deposit are briefly described in a scientific communication by Pinto-Vásquez (1993). The 118 zinc values of de Wijs (1951) are for channel samples cut at 2-m intervals across the steeply dipping Tajo vein along a horizontal mining drift on the 446-m level. This level depth was measured downward from elevation of the San León Tunnel (Fig. 2). The $2.7 \mathrm{~km}$ long Tajo vein was discovered in 1883 and mined until 1956. According to Ahlfield (1954), this "silver mine" had the largest annual zinc and second largest annual silver production in Bolivia. On average, the Tajo vein was $1.10 \mathrm{~m}$ thick with ore containing $14 \% \mathrm{Zn}$ and $0.1 \% \mathrm{Ag}$. Relative sphalerite (zinc sulphide) content increased downward in the orebody. According to Turneaure (1971) the age of the Tajo vein was Neogene, 


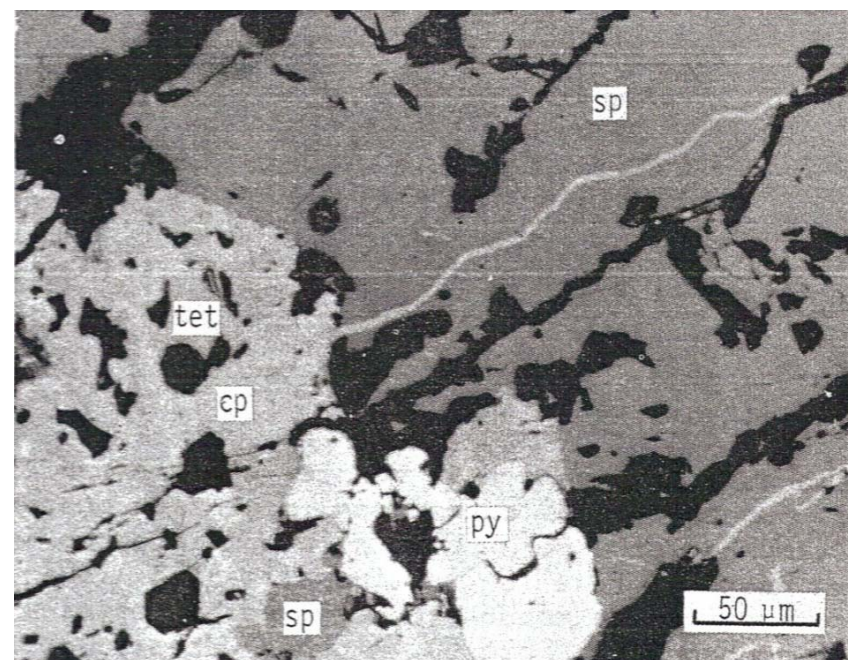

Fig. 3. Micrograph of massive sulphide ore in Pulacayo Mine (from Villalpando and Ueno, 1987). Ore minerals are sphalerite (sp), tetrahedrite (tet), chalcopyrite (cp) and pyrite (py).

probably as young as Pliocene. Figure 3 shows ore minerals at microscopic scale. The silver was in the form of fine grains associated with tetrahedrite. In a conference report by Villalpando and Ueno (1987) it can be seen that zinc content of sphalerite varied between $65.62 \%$ and $66.03 \%$. This implies that maximum possible zinc content of ore consisting exclusively of sphalerite would be $66 \%$ and this is above the largest value of $39.3 \%$ in our data set. However, because the sampled material consisted not only of massive sulphide but also out of mineralized wall rock, the largest possible value is probably considerably less than $66 \%$. This upper limit constrained maximum zinc enrichment.

On the 446-m level, average thickness of massive vein filling averaged only $0.50 \mathrm{~m}$ in width but wall rocks on both sides contained disseminated sphalerite, partly occurring in subparallel stringers. The channel samples were cut over a standard length of $1.30 \mathrm{~m}$, corresponding to expected stoping width. Consequently, each assay value represents average weight percentage zinc for a rod-shaped channel sample of $1.30 \mathrm{~m}$ cut perpendicular to the vein (Fig. 4). The method used for smoothing the 118 zinc values in Fig. 1 was described in Agterberg (1974), who assumed that each zinc value was the sum of a "signal" value and small-scale "noise" with the autocorrelation function $\rho_{h}=c \exp (-a h)$, where $c$ represents the small-scale noise variance and the parameter $a$ controls the decreasing influence of observed values on their surroundings. The two parameters were estimated to be $c=0.5157$ and $a=0.1892$. Signal+noise models of this type are well-known in several branches of science (cf. Yaglom, 1962). Filtering out the noise component produced the signal shown in Fig. 1. Various other statistical methods such as simple moving averaging, kriging or inverse distance weighting can be used to produce similar smoothed patterns.

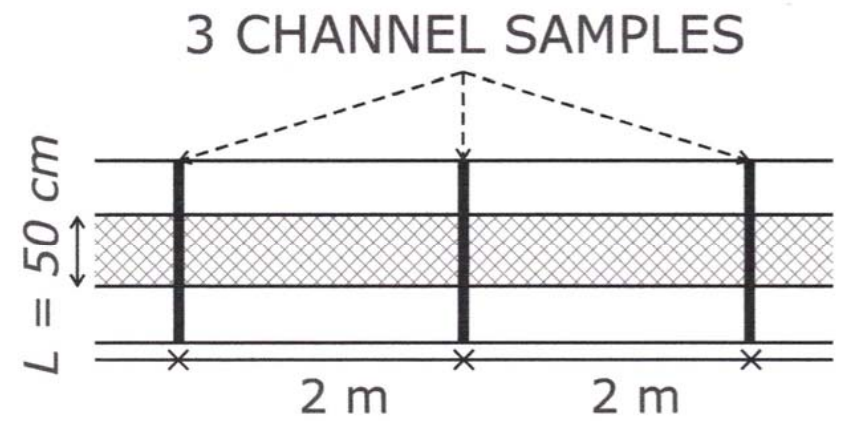

Fig. 4. Schematic representation of channel sampling in Pulacayo Mine. Successive channel samples along horizontal mining drift on 446-m level were $1.3-\mathrm{m}$ long and 2-m apart. The Tajo vein, which is $0.5-\mathrm{m}$ wide on average, consists of massive sulphide (hatched pattern) but wall rock on both sides of the vein contained disseminated sulphide and stringers of sulphide ore. Anticipated stoping width was 1.3-m but "effective" channel sample width $(L)$ was set equal to width of vein $(=0.5 \mathrm{~m})$. Lag distance $(h)$ is $2 \mathrm{~m}$ or multiple of $2 \mathrm{~m}$.

Negative exponential autocorrelation functions are closely related to Markov chain analysis and to scaling properties of sequences of mineral grains in igneous rocks. For example, Xu et al. (2007) demonstrated existence of small-scale scaling in "ideal granite" grain sequences previously modeled as Markov chains (Vistelius et al., 1983). Both continuous and discrete first-order Markov processes have negative exponential autocorrelation functions (Yaglom, 1962; Agterberg, 1974, p. 332). Wang (2008) applied multifractal and Markov chain analysis to sphalerite banding at the microscopic scale in the Jinding lead-zinc deposit, Yunnan Province, China. Larger scale examples of this kind of modeling as applied to lithological data and copper concentration values observed in the KTB deep continental crust drill-hole will be discussed later in this paper.

From the fact that average zinc content on the 446-m level differs from that reported for the entire Tajo vein, it can be assumed that there exist "trends" in the Pulacayo orebody. Agterberg (1961) estimated the amplitude of a sine function fitted to the first 65 of the 118 zinc values, but his best-fitting amplitude of $2.77 \% \mathrm{Zn}$ is not statistically significant. However, it remains possible that there are systematic geographic variations in zinc content within the data set of Fig. 1. This should be kept in mind when spectral analysis is performed later in this paper (Sect. 5.3) because sinusoidal trends would result in over-estimation of low-frequency power densities.

\subsection{Effect of logarithmic transformation}

Matheron (1968) applied geostatistical methods to logarithmically transformed assay values. This can have advantages with respect to using untransformed element concentration values. He assumed that "effective length" of each channel sample could be set equal to $L=0.5 \mathrm{~m}$, representing the 
average width of the Tajo vein on the 446-m level. Obviously, the 118 zinc values of Fig. 1 systematically underestimate true zinc content of the massive sulphide vein filling because the original sample length was $1.30 \mathrm{~m}$ for the massive vein augmented by lower zinc grade wall rocks. Later in this paper, the effective length is assumed to be a variable parameter that can be estimated from observations. In the absence of more complete information, it is not unreasonable to assume, as Matheron did, that all massive sulphide zinc concentration values were underestimated by the same factor during the channel sampling. The logarithmic variance $\sigma^{2}(\ln x)$ is not affected if this bias factor is constant. For our example, $\sigma^{2}(\ln x)$ is estimated to be 0.2851 . One relatively simple geostatistical sampling method can be illustrated as follows. Suppose that the 118 values for channel samples that are $2 \mathrm{~m}$ apart together provide an estimate of average zinc content $(=15.61 \%)$ of an elongated rod-shaped mining block with a length of $238 \mathrm{~m}$. Dividing this number by $L=0.5 \mathrm{~m}$ and raising the quotient to the power 3 then yields $V / v=476$. Combining this number with our estimate of logarithmic variance and using Matheron's equation $\sigma^{2}(\ln x)=\alpha \ln \{V / v\}$ then yields the absolute dispersion estimate $\alpha=0.015$, which would apply to other block sizes as well.

If the logarithmic variance of element concentration values is relatively large, it may not be easy to obtain reliable estimates of statistics such as mean, variance, autocorrelation function and power spectrum by using untransformed element concentration values. However, lognormality of the frequency distribution often can be assumed. This is the main reason for using logarithmically transformed values instead of original values. Suppose that element concentration values can be described by $X_{i}$ and $Y_{i}=\ln X_{i}$ has normal, Gaussian frequency distribution with mean $\mu$ and variance $\sigma^{2}$. Representing the autocorrelation functions for $X_{i}$ and $Y_{i}$ as $\rho_{x}(h)$ and $\rho_{y}(h)$, respectively, we have:

$\sigma^{2} \rho_{y}(h)=\ln \left[1+\gamma^{2} \rho_{x}(h)\right]$

where $\gamma^{2}=\sigma^{2}(X) / \mu^{2}(X)$ (cf. e.g. Agterberg, 1974, Eq. 10.40). If $\gamma^{2}$ is sufficiently small, $\rho_{x}(h)$ and $\rho_{y}(h)$ are approximately equal. For our example, this condition is satisfied as demonstrated in Fig. 5. Approximate equality of results shown in Fig. 5 applies to both the estimated autocorrelation coefficients and negative exponential functions fitted by non-linear least squares to data points with $h>0$. Consequently, variograms of zinc values and logarithmically transformed zinc values also are approximately the same. Later in this paper the variogram of logarithmically transformed zinc values will be used. Substituting fitted values from Fig. 5 into $\gamma(h)=\sigma^{2}\left(1-\rho_{h}\right)$ yields a variogram (Table 1 , see later) that is close to estimates originally obtained by Matheron (1964).

If $\mu$ represents mean of lognormally distributed values, mean and variance of untransformed data satisfy the equations:

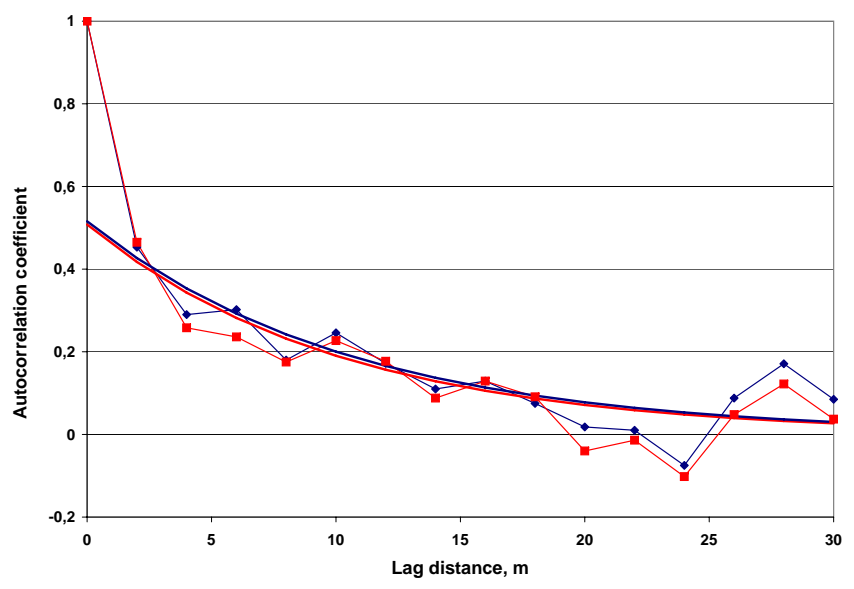

Fig. 5. Estimated autocorrelation coefficients for original data (blue diamonds) and logarithmically transformed zinc values (red squares), shown together with best-fitting negative exponential autocorrelation functions for original data (blue curve) and logarithmically transformed data (red curve), respectively. Patterns are similar illustrating that logarithmic transformation of original data does not significantly affect autocorrelation in this application to the Pulacayo zinc values.

$\mu(X)=e^{\mu+\frac{\sigma^{2}}{2}} ; \sigma^{2}(X)=e^{2 \mu+2 \sigma^{2}}-e^{2 \mu+\sigma^{2}}$

Estimates for our example are $\mu=2.6137$ and $\sigma^{2}=$ 0.2851. Estimates according to Eq. (2) then are $\mu(X)=$ 15.74 and $\sigma^{2}(X)=81.74$. The untransformed zinc values have mean and variance equal to 15.61 and 64.13 , respectively. The larger variance estimate resulting from Eq. (2) may indicate a slight departure from lognormality (largevalue tail slightly weaker than lognormal) possibly related to the fact that the largest possible zinc value is significantly less than $66 \%$ (see before).

\subsection{Other applications of model of de Wijs to ore deposits}

We consider the question of how representative our relatively small, historical data set of 118 zinc values is of ore deposits in general. Matheron (1964) used several other mineral deposits exemplifying his extension of the model of the Wijs. His primary examples were from the Mounana uranium deposit, Gabon, and the Mehengui bauxite deposit, Guyana. These two deposits occurred relatively close to the Earth's surface and were explored by means of subvertical boreholes drilled on regular grids. His other examples included the Bou-Kiama, Montbelleux, Laouni, Mpassa, and Brugeaud orebodies. In all these situations, the model of de Wijs proved to be satisfactory. Some of these examples and others also were discussed in later geostatistical textbooks including David (1977) and Journel and Huijbregts (1978). Later, however, this type of modeling became de-emphasized, probably because the model of de Wijs does not allow for sills that 
Table 1. Pulacayo Mine variogram model, Experimental values from Matheron (1962, p. 180); Lag distance (h) in m; Exponential values from model of Fig. $6 ; f(q)$ as in Eq. (4); $\beta(h)=$ Experimental value $/ f(q) ; \sigma_{h}^{2}=\beta \times f(q)$ is extension variance of $50 \mathrm{~cm}$ line segments; Deviation is difference between colums 2 and 6 . The small deviations indicate good fit of Matheron's variogram model.

\begin{tabular}{rrrrrrr}
\hline$h, \mathrm{~m}$. & Experimental & Exponential & $f(q)$ & $\beta(h)$ & $\sigma_{h}^{2}$ & Deviation \\
\hline 2 & 0.303 & 0.325 & 2.891 & 0.105 & 0.286 & 0.017 \\
4 & 0.402 & 0.367 & 3.580 & 0.112 & 0.354 & 0.048 \\
6 & 0.436 & 0.401 & 3.985 & 0.109 & 0.394 & 0.042 \\
8 & 0.465 & 0.429 & 4.273 & 0.109 & 0.422 & 0.043 \\
10 & 0.408 & 0.452 & 4.496 & 0.091 & 0.444 & -0.036 \\
12 & 0.412 & 0.471 & 4.678 & 0.088 & 0.462 & -0.050 \\
14 & 0.464 & 0.486 & 4.832 & 0.096 & 0.477 & -0.013 \\
16 & 0.452 & 0.499 & 4.966 & 0.091 & 0.491 & -0.039 \\
18 & 0.472 & 0.510 & 5.083 & 0.093 & 0.502 & -0.030 \\
20 & 0.545 & 0.518 & 5.189 & 0.105 & 0.513 & 0.032 \\
\hline
\end{tabular}

occur generally and problems associated with working with logarithmically transformed concentration values instead of original data. However, as pointed out by Matheron (1974), lognormality is an issue that must be considered generally. Multifractal modeling (e.g. use of multiplicative cascades) confirms the validity of several aspects of Matheron's original approach. The multifractal autocorrelation function of Cheng and Agterberg (1996) has a sill as well as a nugget effect with exceptionally strong autocorrelation over very short distances (cf. Sect. 4.2).

Agterberg (1965) estimated autocorrelation coefficients for the original de Wijs zinc data and obtained similar results for titanium data from adjoining borehole samples in a magnetite deposit, Los Angeles County, California, originally described by Benson et al. (1962). Later in this paper, this example will be used for comparison with spectral analysis results for the de Wijs zinc data. Figure 6a (modified from Agterberg, 1974, Fig. 56) shows average autocorrelation coefficients and best-fitting negative exponential function derived from logarithmically transformed element concentration values for copper from the Whalesback copper deposit, Newfoundland, and Fig. $6 \mathrm{~b}$ and c are for two relatively long series of gold assays from the Orange Free State Mine, Witwatersrand goldfields, South Africa (data from Krige et al., 1969). In these three examples, the negative exponential function with significant noise component provides a good fit. In each situation, there is finite variance (existence of sill) and a de Wijsian variogram can only be fitted for the copper and gold examples over relatively short distances (for approximately the first six values from the origin in the three examples of Fig. 6).

A typical sample of 1090 copper concentration values from the Whalesback deposit (cf. Agterberg, 1974, p. 301) had mean value of $1.57 \% \mathrm{Cu}$ and logarithmic variance of 1.21. Substitution of these values into Eq. (2) yields $\mu=$ 0.857 and $\sigma^{2}(X)=43.84$. The positive skewness of the copper concentration is so large that it is not possible to obtain reliable statistics from original data without use of a more efficient estimation method involving logarithmic transformation (Aitchison and Brown, 1957; Sichel, 1966). The logarithmic variance of the gold values in the other example is approximately 1.03. Krige et al. (1960) do not report the corresponding mean value but the following statistics can be derived from a relatively small data set of 61 gold values in Krige and Ueckermann (1963): $\mu(X)=906.6$; $\sigma^{2}(X)=1470410 ; \mu=6.134$; and $\sigma^{2}=0.929$. Substitution of the latter two estimates into Eq. (2) yields new estimates of $\mu(X)=879.1 ; \sigma^{2}(X)=1183972$. In this application, the new estimates are probably better than those obtained from the original gold values without use of an appropriate transformation.

The comparison of the Pulacayo zinc example with the Whalesback copper and Witwatersrand gold examples illustrates that there are similarities in that the frequency distributions of channel samples in all three examples are positively skewed and approximately lognormal. Also, in all three cases, the autocorrelation function can be approximated by a negative exponential function with value less than unity at the origin indicating existence of a noise component superimposed on the spatial random variable representing more continuous variability at larger distances. In Fig. 1 the noise component was filtered out to retain a "signal" with approximately unity autocorrelation function value at the origin (cf. Agterberg, 1974). The nugget effect can be modeled as random noise at lag distances greater than $2 \mathrm{~m}$. However, as originally realized by Matheron (1971), a nugget effect of this type may reflect strong autocorrelation so close to the origin that it cannot be seen in semivariograms or correlograms because its spatial extent is less than the sampling interval used in practice. The frequency distribution of the Pulacayo zinc example has less positive skewness than those of copper and gold in the other examples. Also, existence of a sill is not obvious in the Pulacayo zinc example. 

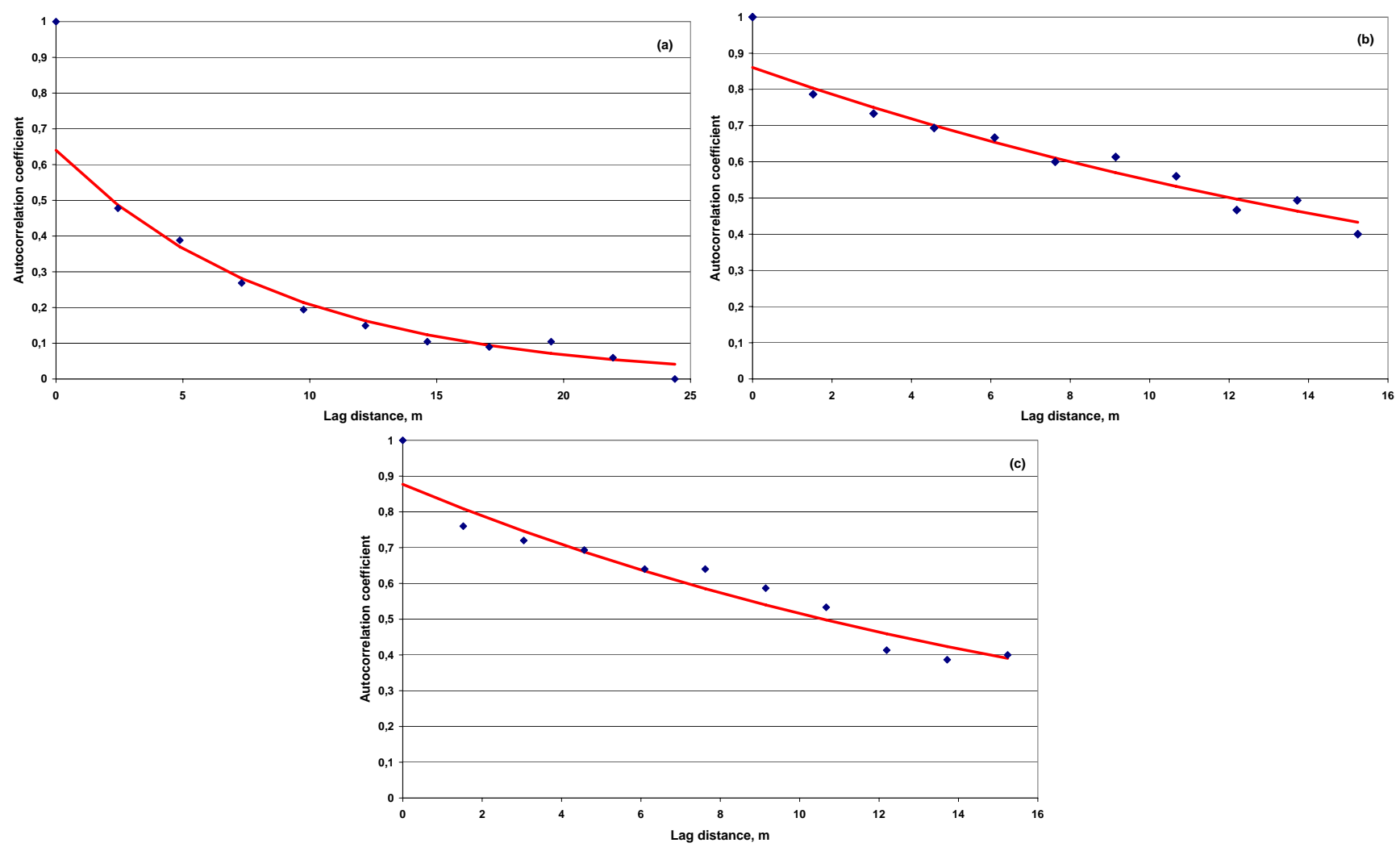

Fig. 6. Estimated autocorrelation coefficients (blue diamonds) and best-fitting negative exponential autocorrelation functions (red curves) derived from logarithmically transformed element concentration values: (a) Average of correlograms for 24 channel sample series from drifts at various levels of Whalesback copper deposit, Newfoundland (after Agterberg, 1974); (b) Series of 462 gold assays from the Orange Free State Mine, Witwatersrand goldfields, South Africa (modified from Krige et al., 1969); (c) Other series of 540 gold assays from same gold mine. In each diagram the fitted exponential (red line) intersects the vertical axis at a point with autocorrelation coefficient less than 1 indicating the existence of a nugget effect.

\section{Geometrical probability modeling for model of de Wijs}

Matheron (1962) showed that the semivariogram $\gamma(h)$ in his extension of the model of de Wijs satisfies:

$\gamma(h)=3 \alpha \times \ln h$

where, as before, $\alpha$ is absolute dispersion. In principle, this model also can be applied to untransformed data. The following applications of geometrical probability are for logarithmically transformed distance.

Suppose that $\mathrm{AA}^{\prime} \mathrm{BB}^{\prime}$ represents a rectangle with sides $\mathrm{AA}^{\prime}=\mathrm{BB}^{\prime}=h, \mathrm{AB}=\mathrm{A}^{\prime} \mathrm{B}^{\prime}=L$ and $\tan \theta=L / h$ (Fig. 7). If the concentration value for a small volume at a point is taken to be the concentration value of another volume of rock that either contains the small volume or is located elsewhere, this results in uncertainty expressed by means of the "extension variance". In Matheron (1962, Sect. 39) or Agterberg (1974, Sect. 10.11) it is discussed in detail that the variogram value of parallel line segments of length $L$ that are distance $h$ apart

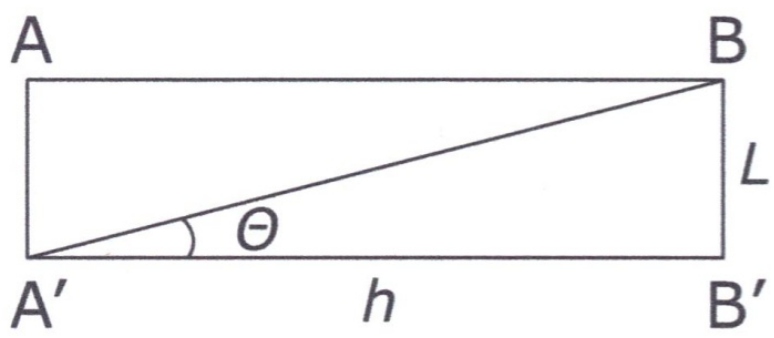

Fig. 7. Graphical illustration of relationship between $\theta$, effective length $(L)$, and lag distance $(h)$.

along a straight line can be interpreted as an extension variance $\sigma_{h}^{2}=\beta f(\theta)$ with $\beta=6 \alpha$ and:

$$
f(\theta)=-\ln \frac{L}{\sqrt{L^{2}+h^{2}}}+\frac{2 h}{L} \tan ^{-1} \frac{L}{h}+\frac{h^{2}}{L^{2}} \ln \frac{h}{\sqrt{L^{2}+h^{2}}}
$$

Table 1 shows the first 10 Pulacayo Mine variogram values as estimated by Matheron (1962, p. 180) using Eq. (4) for logtransformed (base e) zinc values. For comparison, theoretical 


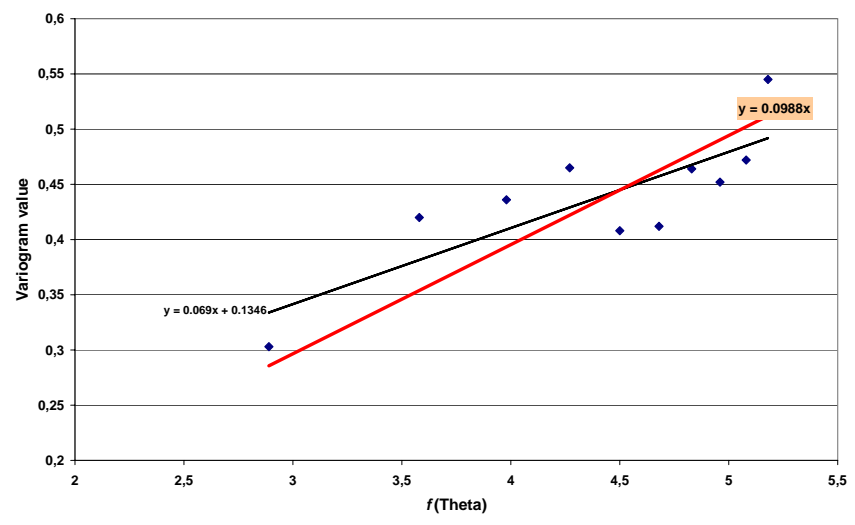

Fig. 8. Straight line (red) for Pulacayo zinc values fitted by constrained least squares to 10 variogram values taken from Matheron (1964). Horizontal axis is for $f(\theta)$. Line was forced through the point where $f(\theta)=0$ and $h=0$ (cf. Eq. 4). Its slope $\beta=0.0988$ yields estimate of absolute dispersion $\alpha(=0.0165)$. Best-fitting line without constraint (black), that is significantly different, is shown for comparison.

variogram values for the exponential model (derived from autocorrelation model graphically shown in Fig. 5) are listed as well, illustrating that this model with a sill also provides a good fit. For other theoretical autocorrelation functions fitted to the Pulacayo zinc values, see Sect. 4.2 and Chen et al. (2007).

Equation (4) resulted in multiple estimates of $\beta(h)$ for different lag distances $(h)$ in Table 1. A better estimate is obtained by using constrained least squares estimation as follows. The theoretical variogram values in the second last column of Table 1 are based on a single estimate $(\beta=0.0988)$ representing the slope of a line of best fit (Fig. 8) forced through the point where $f(\theta)$ and $h=0$. This additional point receives relatively strong weight in the linear regression because it is distant from the cluster of the other 10 points used. The constraint can be used because, for decreasing $h$ :

$\lim _{h \rightarrow 0} f(\theta)=-\lim _{h \rightarrow 0}\left\{\ln \frac{L}{\sqrt{L^{2}+h^{2}}}\right\}+\lim _{h \rightarrow 0}\left\{\frac{2 h}{L} \tan ^{-1} \frac{L}{h}\right\}+$

$\lim _{h \rightarrow 0}\left\{\frac{h}{\sqrt{L^{2}+h^{2}}}\right\}=0$

The new estimate of absolute dispersion $\alpha=\beta / 6=0.0165$ not only produces theoretical variogram values, which are nearly equal to the estimates based on the logarithmically transformed zinc values, it also is nearly equal to $\alpha=0.015$ previously derived from the logarithmic variance in the previous section, confirming the applicability of Matheron's original method within a neighbourhood extending from about $2 \mathrm{~m}$ to $400 \mathrm{~m}$.

Matheron's geometrical approach can be used for several other purposes. For example, in applications of multifractal modeling to the Pulacayo Mine (Cheng and Agterberg, 1996; Chen et al., 2007; Lovejoy and Schertzer, 2007), it is

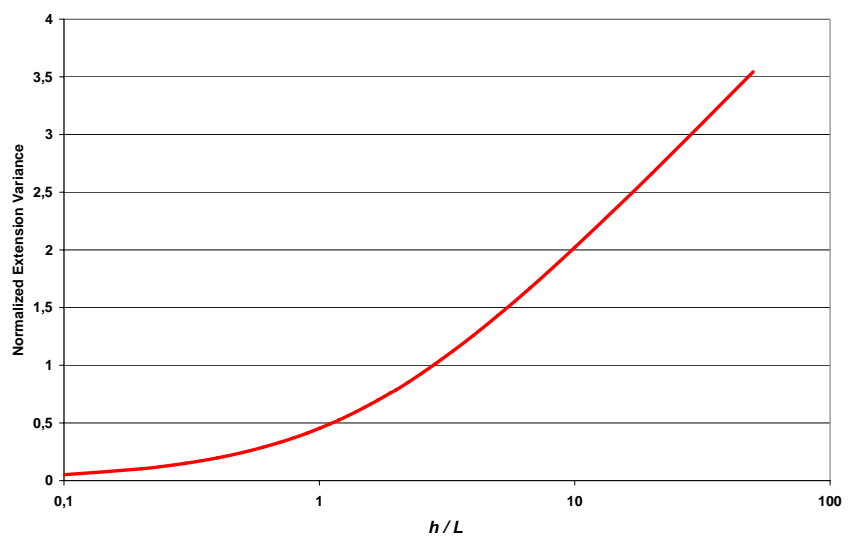

Fig. 9. Relationship between normalized extension variance $\left(\sigma_{\mathrm{E}}^{2}\right)$ and $h / L$.

assumed that the zinc concentration values can be converted into measures of amounts of zinc in adjoining $2 \mathrm{~m}$ wide samples along a line parallel to the drift on the 446-level. It implies that every zinc concentration value for a channel sample at a point along this line is taken as representative for a width of $2 \mathrm{~m}$. Associated uncertainty then is given by the extension variance $\sigma_{\mathrm{E}}^{2}$. Figure 9 shows that normalized extension variance $\sigma_{\mathrm{E}}^{2} / 3 \alpha$ depends on $h / L$. From our estimate $\alpha=0.0165$, it follows that $\sigma_{\mathrm{E}}^{2}=0.0622$ for $h=2 \mathrm{~m}$ wide samples that are $L=50 \mathrm{~cm}$ long. It probably significantly overestimates true value because absolute dispersion is less than 0.0165 over very short distances due to the nugget effect (see later). If $\alpha<0.0165$, the normalized extension variance is greater than $\sigma_{\mathrm{E}}^{2}=0.0622$ as derived for the same value of $h / L$ from the curve in Fig. 9, that is for $\alpha=0.0165$.

Matheron (1964) has shown that the average of $n$ adjoining channel sample concentration values has variance equal to $\sigma_{\mathrm{E}}^{2} / n$. This is another important result because, in Section 5, average values with $n$ equal to $3,5,7$, and 9 will be used extensively. The extension variance $\sigma_{\mathrm{E}}^{2}=0.0622$ is for logarithmically transformed zinc concentration values. As discussed in Sect. 2.2, it can be assumed that the zinc values ( $X_{i}$ with $\left.i=1, \ldots, 118\right)$ for the original channel samples systematically underestimate zinc values for the massive sulphide (Fig. 4). By setting $\sigma^{2}=\sigma_{\mathrm{E}}^{2}$ and $\mu(X)=X_{i}$ in Eq. (2), it is possible to estimate the variances $\sigma^{2}\left(X_{i}\right)$ of the original zinc values. These variances can then be used to calculate approximate $95 \%$ confidence limits for zinc concentration values of $1.3 \mathrm{~m} \times 2 \mathrm{~m}$ plates formed by extending the $1.3 \mathrm{~m}$ long channel samples by $1 \mathrm{~m}$ on both sides. Table 2 shows $\pm 1.96 \sigma\left(X_{i}\right)$ error bars for 11 original zinc values and for averages of adjacent values for wider plates at the same locations. These sets of overlapping plates, that are $20 \mathrm{~m}$ apart, were selected for example so that both low and high zinc concentrations are represented. The error bars in Table 2 for plates wider than $2 \mathrm{~m}$ are relatively narrow. Uncertainty is greatest for the $1.3 \mathrm{~m} \times 2 \mathrm{~m}$ plates but this is probably 
because $\alpha=0.0165$ is underestimated over very short distances resulting in error bars that are too wide (see previous paragraph). The problem of overestimation of extension variances of average element concentration values for small plates due to local strong autocorrelation was previously considered by Matheron (1989, p. 73-75) as follows. Ten professional geostatisticians were provided with a set of variogram values with unit of lag distance equal to $180 \mathrm{~m}$. Independently the participants in this experiment were asked to (a) fit a variogram, and (b) calculate the corresponding extension variance for a square plate measuring $180 \mathrm{~m}$ on a side. Each variogram fitted by a participant had a nugget effect, with, in addition, an exponential or (third-order polynomial) "spherical" variogram curve. The corresponding average of ten estimated extension variances was $0.4019 \pm 0.0127$ indicating excellent agreement between participants. Next, the same 10 people were provided with additional variogram values for shorter unit lag distance interval of $20 \mathrm{~m}$. Again they were asked (a) fit a variogram, and (b) calculate the corresponding extension of the $180 \mathrm{~m} \times 180 \mathrm{~m}$ square plate. The variogram models used during the second stage of the experiment were "richer" becoming either: nugget + spherical + spherical, or nugget + exponential + spherical, or nugget + exponential + exponential. A few other answers were given as well. The revised average extension variance became $0.3686 \pm 0.0062$. Clearly this revised estimate of the extension variance is less than the first estimate and outside the $95 \%$ confidence of the first estimate. Similar results were obtained during a third stage of this experiment using an even shorter unit lag distance.

The preceding experiment illustrates (a) different variogram models applied to the same data sets can produce similar estimates of extension variances; and (b) extension variance estimates are too large if there is a "nugget effect" incorporating strong autocorrelation over very short distances. In the remainder of this paper it will be attempted to model this type of nugget effect by (a) extrapolation from the original variogram values, (b) multifractal modeling, and (c) spectral analysis. The Pulacayo zinc example will be re-analyzed. Because this series is based on 118 values only, the estimated autocorrelation (or variogram) values have limited precision as previously shown by Agterberg $(1965,1967)$. For this reason, autocorrelation for a very large data set was studied as well. II will be shown that there is a nugget effect in copper concentration values from along the deep KTB borehole with short-distance extent that is similar in consecutive series of 1000, 1000 and 796 values, respectively.

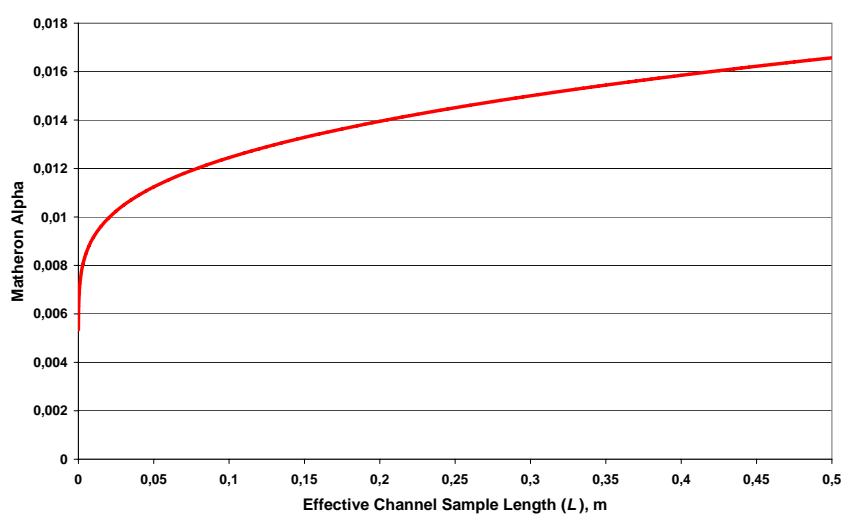

Fig. 10. Constrained least squares estimation of $\beta$ for $L=0.5 \mathrm{~m}$ (see Fig. 8) repeated for effective channel sample lengths less than $0.5 \mathrm{~m}$. Variogram and autocorrelation functions fitted for distances $h>2 \mathrm{~m}$, where absolute dispersion is $\alpha=0.0165$, lose validity over shorter distances due to the nugget effect, that results in a decrease in Matheron's $\alpha$, especially when $L<0.03 \mathrm{~m}$. At the microscopic scale $(h<0.003 \mathrm{~m}$, cf. Fig. 3), $\alpha$ can be expected to increase rapidly again.

\section{Nugget effect and local singularity analysis}

\subsection{Strong autocorrelation and decorrelation over very short distances}

In Sect. 2, it was pointed out that there is uncertainty associated with the definition of effective length $L=0.5 \mathrm{~m}$ of the channel samples in the Pulacayo Mine. This is because these samples were taken across entire width $(=1.30 \mathrm{~m})$ of drift whereas Tajo vein has (horizontally measured) thickness of $0.50 \mathrm{~m}$ on the $446-\mathrm{m}$ level. This thickness value was used by Matheron and earlier in this paper as a best estimate of $L$. It has been shown that the choice of $L=0.5$ results in estimates of $\alpha$ that are satisfactory for lag distances greater than $2 \mathrm{~m}$ (up to $400 \mathrm{~m}$ ). For shorter lag distances, however, it is useful to generalize Matheron's concept of absolute dispersion by defining $\alpha(L)$, which depends on the value of $L$. Consequently, $\alpha=\alpha(0.5)$ for the applications described in Sect. 3. Theoretically, the method used to estimate $\beta(0.5)=6 \alpha(0.5)$ in Fig. 8 can be used to optimize our choice of $L$. Figure 10 shows estimates of $\alpha(L)$ that would be obtained for effective channel sample lengths less than $0.5 \mathrm{~m}$. For $L>3 \mathrm{~cm}, \alpha(L)$ increases slightly from about 0.01 to 0.0165 at $L=0.5 \mathrm{~m}$; for $L<0.03 \mathrm{~m}$, there is rapid decrease to $\alpha(L)=0$. Figure 11 shows sum of squared deviations from lines of best fit for different values of $L$. The optimum solution $(\alpha(L)=0.021)$ is obtained at $L=13 \mathrm{~cm}$. The de Wijsian variogram model that best fits the 10 observed values of Table 1 is for linear samples that are not only shorter than the channel samples on which zinc concentration was measured $(L=1.3 \mathrm{~m})$ but also shorter than the thickness of the Tajo vein $(L=0.5 \mathrm{~m})$. This results probably reflects small-scale clustering of the chalcopyrite crystals. It should 
Table 2. Zinc concentration values (in \%) with $95 \%$ confidence intervals for thin plates elongated in the direction of themining drift with channel samples at their centers. Results are shown for every 10th value in the original seriesof 118 Pulacayo zinc values. Error bars for $1.3 \mathrm{~m} \times 2 \mathrm{~m}$ are too wide because small-scale spatial correlationis not being considered.

\begin{tabular}{rccccc}
\hline Plate size & $1.3 \mathrm{~m} \times 2 \mathrm{~m}$ & $1.3 \mathrm{~m} \times 6 \mathrm{~m}$ & $1.3 \mathrm{~m} \times 10 \mathrm{~m}$ & $1.3 \mathrm{~m} \times 14 \mathrm{~m}$ & $1.3 \mathrm{~m} \times 18 \mathrm{~m}$ \\
\hline$\# 10$ & $24.1 \pm 12.2$ & $19.9 \pm 5.6$ & $19.4 \pm 4.2$ & $17.5 \pm 3.2$ & $17.0 \pm 2.8$ \\
$\# 20$ & $15.1 \pm 7.7$ & $13.8 \pm 3.9$ & $14.0 \pm 3.1$ & $13.3 \pm 2.4$ & $13.2 \pm 2.1$ \\
$\# 30$ & $9.5 \pm 4.8$ & $12.1 \pm 3.4$ & $15.2 \pm 3.3$ & $13.2 \pm 2.4$ & $14.7 \pm 2.4$ \\
$\# 40$ & $10.6 \pm 5.4$ & $15.6 \pm 4.4$ & $17.0 \pm 3.7$ & $15.5 \pm 2.9$ & $14.2 \pm 2.3$ \\
$\# 50$ & $27.4 \pm 13.9$ & $18.6 \pm 5.3$ & $17.4 \pm 3.8$ & $17.4 \pm 3.2$ & $17.2 \pm 2.8$ \\
$\# 60$ & $4.7 \pm 2.4$ & $9.0 \pm 2.5$ & $8.7 \pm 1.9$ & $8.1 \pm 1.5$ & $9.0 \pm 1.5$ \\
$\# 70$ & $9.7 \pm 4.9$ & $9.2 \pm 2.6$ & $10.5 \pm 2.3$ & $10.3 \pm 1.9$ & $10.2 \pm 1.6$ \\
$\# 80$ & $10.6 \pm 5.4$ & $11.1 \pm 3.2$ & $10.8 \pm 2.3$ & $9.3 \pm 1.7$ & $9.6 \pm 1.6$ \\
$\# 90$ & $30.8 \pm 15.6$ & $31.6 \pm 9.0$ & $30.8 \pm 6.7$ & $30.7 \pm 5.7$ & $29.2 \pm 4.7$ \\
$\# 100$ & $22.6 \pm 11.5$ & $16.4 \pm 4.6$ & $18.6 \pm 4.1$ & $20.8 \pm 3.8$ & $21.4 \pm 3.5$ \\
$\# 110$ & $7.9 \pm 4.0$ & $17.8 \pm 5.0$ & $17.2 \pm 3.8$ & $15.9 \pm 2.9$ & $14.6 \pm 2.4$ \\
\hline
\end{tabular}

be kept in mind that this conclusion remains subject to uncertainty because of limited precision of the variogram values of Table 1. Also, anisotropy may have played a role because zinc concentration value variability perpendicular to the Tajo vein could well differ from variability parallel to the vein. However, the best explanation is that over short lag distances $h$ (e.g. within the domain $0.003 \mathrm{~m}<h<2 \mathrm{~m}$ ) there exists a strong nugget effect that is not readily detectable at distances of $h \geq 2 \mathrm{~m}$. At the microscopic level we would expect $\alpha$ to increase rapidly again, because of measurement errors and the fact that the zinc occurs in sphalerite crystals only (cf. Fig. 3). The crystal boundary effect may have become negligibly small in our application because channel sample length greatly exceeded crystal dimensions.

The preceding considerations imply that the negative exponential autocorrelation function previously used (see e.g. Fig. 5) is too simple for short distances $(h<2 \mathrm{~m})$. The true pattern is probably close to that shown in Fig. 12, which differs from the earlier model in that strong autocorrelation is assumed to exist over very short distances. It is probably caused by clustering of ore crystals, although at the microscopic scale there remains rapid decorrelation related to measurement errors and crystal shapes. The graph in Fig. 12a satisfies the equation:

$p(h)=c_{1} e^{-a_{1} h}+c_{2} e^{-a_{2} h}$

The coefficients in the first term are $c_{1}=0.5157$ and $a_{1}=0.1892$ as in Fig. 5. The second term represents the strong autocorrelation due to clustering over very short distances. The decorrelation at microscopic scale is represented by a small white noise component with variance equal to $c_{0}=0.0208$ as will be determined in Sect. 4.4. The coefficient $c_{2}$ in the second term on the right side of Eq. (6) satisfies $c_{2}=1-c_{0}-c_{1}=0.4635$. Because of lack of more detailed information on autocorrelation over very short distances, it is difficult to choose a good value for the coefficient $a_{2}$. We can

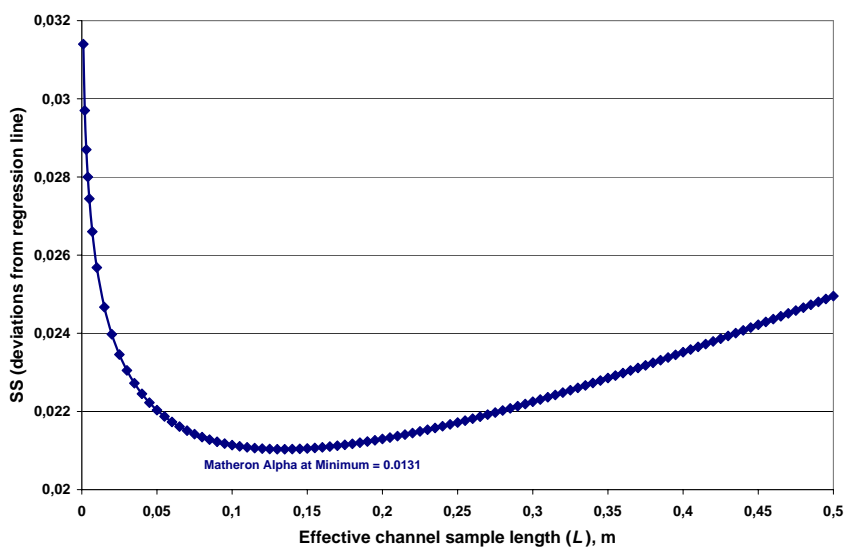

Fig. 11. Sum of squared deviations from lines of best fit as a function of Matheron's $\alpha$ and linear sample length $L$. The rapid increase of this function near $L=0$ probably reflects randomness at the microscopic scale plus white noise due to measurement errors. Optimum effective channel sample width $(L=0.13 \mathrm{~m})$ is based on variogram values with limited precision for the 2 to $20 \mathrm{~m}$ neighbourhood. Because of this, this estimate is subject to uncertainty. It could reflect the fact that the Tajo vein was formed by crystallization of ore minerals outward from a narrow fissure in the surrounding sandstone and conglomerates (cf. Fig. 2).

assume that, approximately, $a_{2}=2$. The choice of this value for $a_{2}$ provides a good fit over the entire observed correlogram (Fig. 5). It affects extrapolation toward the origin with $h<2 \mathrm{~m}$ only. Figure $12 \mathrm{~b}$ shows that the second term on the right side of Eq. (6) cannot be detected in the correlogram for sampling intervals greater than $2 \mathrm{~m}$. Other types of evidence for existence of strong autocorrelation over very short distances in the Pulacayo orebody will be presented in later Sects. (4.2, 5.2, and 6.3, respectively). In the Sect. 4.2, a multifractal autocorrelation function will be derived on the basis 

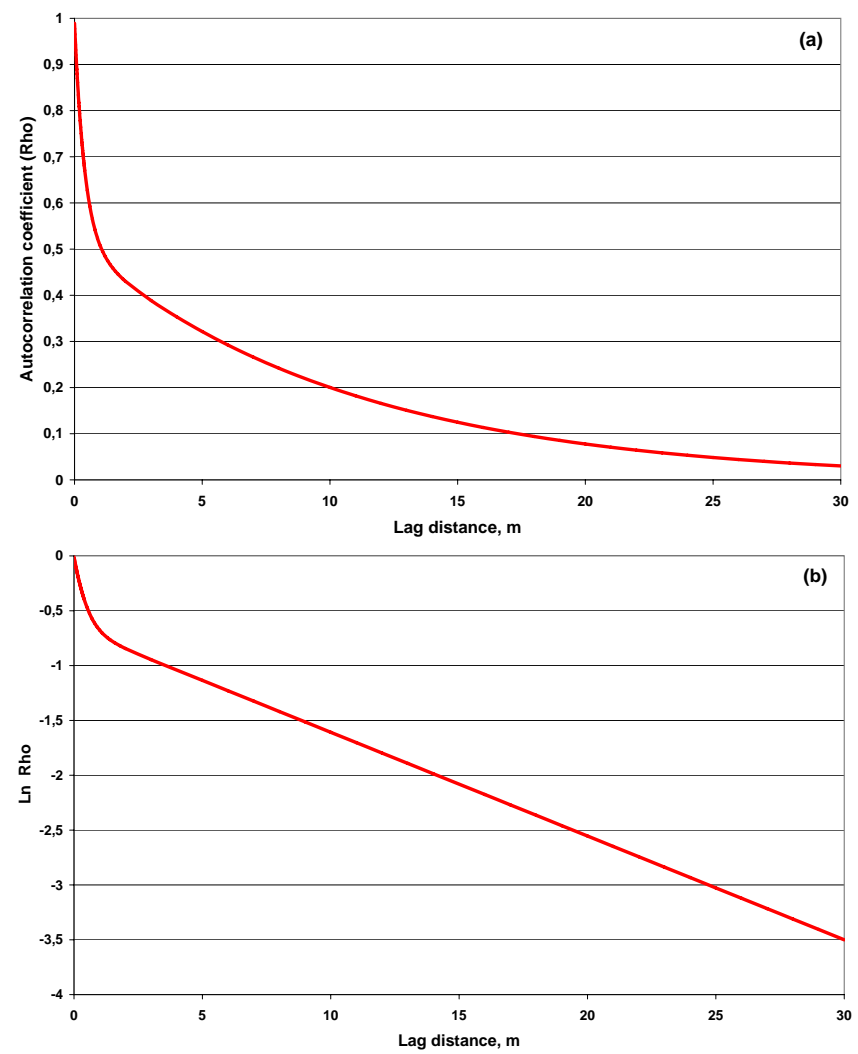

Fig. 12. Hypothetical autocorrelation function consisting of two negative exponential functions to incorporate nugget effect over short lag distances $h$ with $h<2 \mathrm{~m}$. Autocorrelation function for nugget effect is superimposed on negative exponential curve for distances $h>2 \mathrm{~m}$ previously used to filter out the nugget effect in order to retain "signal" of Fig. 1: (a) Graph of Eq. (17); (b) Same graph as in (a) but with logarithmic scale for autocorrelation (Rho).

of self-similarity assumptions. It results in a curve (Fig. 14) that resembles the one based on Eq. (6) (with $a_{2}=2$ ). For example, for lag distance equal to $60 \mathrm{~cm}$, the theoretical value according to Fig. 12a is 0.6 , while Fig. 14 yields 0.7.

\subsection{Theoretical multifractal autocorrelation function}

Cheng and Agterberg (1996) have shown that the series of 118 Pulacayo zinc values can be modeled as a multifractal with "partition function" $\chi_{q}(\varepsilon)$ (cf. Evertsz and Mandelbrot, 1992) for a sequence of "moments" $q$ and line segments of length $\varepsilon$. The unit of $\varepsilon$ was set equal to the sampling interval $(=2 \mathrm{~m})$. The $\log -\log$ plot of $\chi_{q}(\varepsilon)$ versus $q$ shows approximately straight lines for different values of $q$ (Cheng and Agterberg, 1996, Fig. 2a). The slopes of these straight lines provide estimates of the "mass exponents" $\tau(q)$, which can be used to construct the multifractal spectrum (cf. Feder, 1988). Cheng and Agterberg (1996) derived the following expression for the autocorrelation function of a multifractal of this type:

$$
\begin{aligned}
& \rho_{k}(\varepsilon)= \\
& \frac{c \varepsilon^{\tau(2)-2}}{2 \sigma^{2}(\varepsilon)}\left[(k+1)^{\tau(2)+1}-2 k^{\tau(2)+1}+(k-1)^{\tau(2)+1}\right]-\frac{\xi^{2}}{\sigma^{2}(\varepsilon)}
\end{aligned}
$$

where $C$ is a constant, $\varepsilon$ represents length of line segment for which an average zinc concentration value is assumed to be representative, $\tau(2)$ is the second-order mass exponent, $\xi$ represents overall mean concentration value, and $\sigma^{2}(\varepsilon)$ is the variance of the zinc concentration values. The unit interval $\varepsilon$ is measured in the same direction as the lag distance $h$. The index $k$ is an integer value, that later in this section will be transformed into a measure of distance by means of $k=1 / 2 h$. Estimation for the 118 Pulacayo zinc values using an ordinary least squares model with $\tau(2)=0.979$ gave (see Cheng and Agterberg, Eq. 35):

$\hat{\rho}_{k}=4.37\left[(k+1)^{1.979}-2 k^{1.979}+(k-1)^{1.979}\right]-8.00$

The first 15 values $(k \geq 1)$ resulting from Eq. (8) are nearly the same as the best-fitting semi-exponential previously shown in Figs. 6 and 12. The model can be extrapolated toward the origin by replacing the second-order difference on the right side of Eq. (7) by the second derivative:

$\left[(k+1)^{\tau(2)+1}-2 k^{\tau(2)+1}+(k-1)^{\tau(2)+1}\right] \cong\{\tau(2)+1\} k^{\tau(2)-1}$

Linear regression of the second derivative for $\tau(2)=0.979$ on estimated values obtained by means of Eq. (8) then results in the straight-line approximation shown in Fig. 13. Although the largest estimated value of that could be obtained by Eq. (8) is only 0.487 (for $k=1$ ), it becomes possible to extrapolate toward smaller values of $k=1 / 2 h$, so that larger autocorrelation coefficients are obtained, by using the second derivative on the right side of Eq. (9) instead of the second-order difference. The theoretical autocorrelation function shown in Fig. 14 was derived by transformation of the straight line of Fig. 13 for lag distances with $h \geq 0.014 \mathrm{~m}$. For integer values $(1 \leq k \leq 15)$, the curve of Fig. 14 reproduces the estimated autocorrelation coefficients obtained by Eq. (8). Using it for extrapolating toward the origin results in an overall pattern that closely resembles the hypothetical pattern of Fig. 12a consisting of two superimposed negative exponentials with a small white noise component. Consequently, the multifractal autocorrelation model of Cheng and Agterberg (1996), which is based on the assumption of scaleindependence, confirms the existence of strong autocorrelation over short distances $(h<2 \mathrm{~m})$.

\subsection{Comparison to spatial covariance modeling of KTB velocity and lithology logs}

An autocovariance function consisting of two superimposed negative exponentials with different scaling constants originally was obtained by Goff and Holliger (1999) for binary 


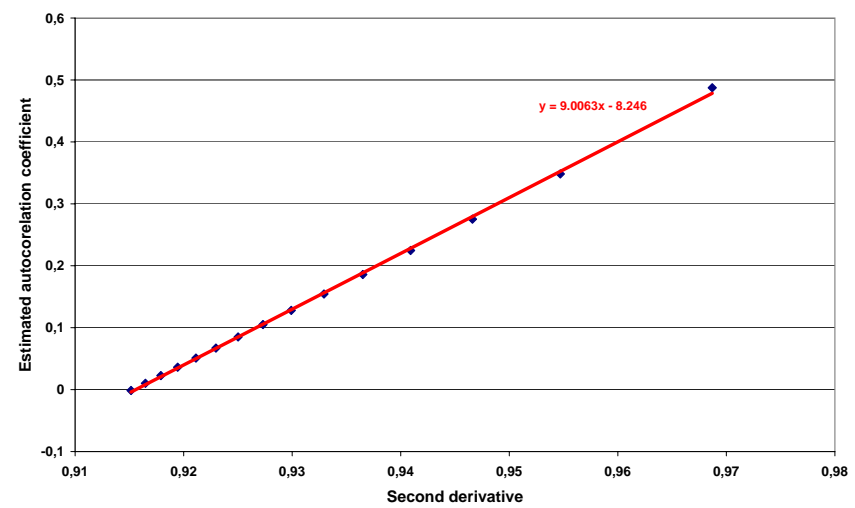

Fig. 13. Relation between estimated autocorrelation coefficients (blue diamonds) and second derivative of corresponding continuous function (Eq. 9). Best-fitting straight line (colored red) will be used for extrapolation to the origin (see Fig. 14).

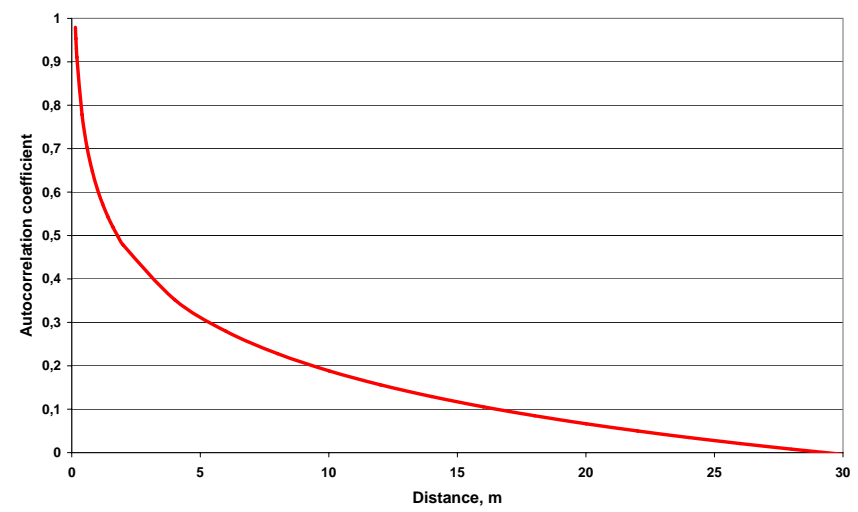

Fig. 14. Theoretical multifractal autocorrelation function derived by using the best-fitting straight line in Fig. 13. For lag distances $h \geq 2 \mathrm{~m}$, autocorrelation coefficients approximately satisfy Eq. (9); for $h<2 \mathrm{~m}$, the model indicates a nugget effect with shape of autocorrelation function resembling hypothetical curve of Fig. 12.

lithology values derived from velocity and lithology logs for the main borehole of the German Continental deep Drilling Program (KTB). In our Fig. 12, $a_{1}=0.1892$ for larger scale variability and $a_{2}=2$ was assumed for nugget effect. In Goff and Holliger's Fig. 7, $a_{1}=0.001$ for the "large scale" and $a_{2}=0.019$ for the "small scale" model. The dimensionless ratio $a_{2} / a_{1}$ for KTB binary lithology is 19 and somewhat greater than our ratio of 11 in Fig. 12. Lithology in the main KTB borehole was determined at points that are $1 \mathrm{~m}$ apart over a length of about $7 \mathrm{~km}$. In general, significant pre-processing is required for the analysis of long series of this type. Goff and Hollinger (1999) commenced this process by plotting raw compressional velocity $\left(V_{p}\right)$ averaged within more or less homogeneous lithological sections against depth. A deterministic component derived from this plot was extracted for the purpose of detrending followed by conversion of the lithology $\log$ into a binary residual $V_{p}$

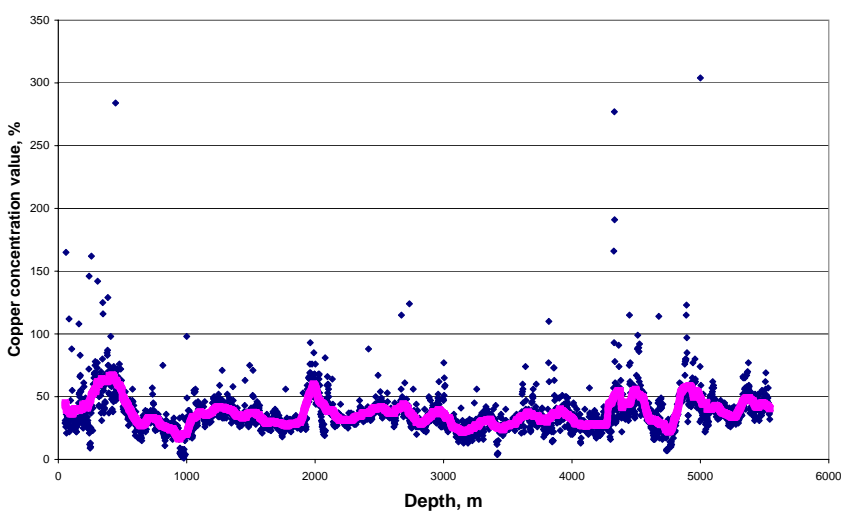

Fig. 15. Copper concentration (ppm) values from Main KTB borehole together with mean values for $101 \mathrm{~m}$ long segments of drillcore. Locally the original data (blue diamonds) deviate strongly from the moving average (pink line).

profile for which the spatial covariance in $\left(\mathrm{km} \mathrm{s}^{-1}\right)^{2}$ was estimated. The two rock types retained in the binary plot are mainly metabasite $\left(V_{p}=+0.2 \mathrm{~km} \mathrm{~s}^{-1}\right)$ and mainly gneiss $\left(V_{p}=-0.2 \mathrm{~km} \mathrm{~s}^{-1}\right)$.

The von Kármán autocovariance model has been used extensively to characterize crustal heterogeneity properties not only for velocity log properties (e.g. Wu and Aki, 1985; Wu et al., 1994; Goff and Hollinger, 1999, 2003) but also for geological maps of crustal exposures (e.g. Goff et al., 1994; Goff and Levander, 1996), seafloor morphology (Goff and Jordan, 1988), and in field simulations (Goff and Jennings, 1999). This model was first proposed by von Kármán (1948) and can be written as:

$\rho(h)=\frac{(a h)^{v} K_{v}(a h)}{2^{v-1} \Gamma(v)}$

where $v$ is the Hurst number (cf. Mandelbrot, 1983; Chemingui, 2001; Klimeš, 2002), and $K_{v}$ is the modified Bessel function of order $v$. Fitting of the two-parameter von Kármán model to an estimated covariance function can be performed using the inversion methodology of Goff and Jordan (1988). If $v=0.5$, Eq. (10) reduces to $\rho(h)=\exp (-a h)$. Goff and Hollinger's (1999) best Eq. (10) von Kármán model fit for the KTB binary residual $V_{p}$ profile has $v=0.21$ and $a=0.00072$. However, a better fit for the autocovariance of this series was obtained by these authors using Eq. (6) with $c_{0}=0, c_{1}=0.684, c_{2}=0.316, a_{1}=0.001$ and $a_{2}=0.019$. The Hurst numbers for both negative exponentials are equal to 0.5 , more than twice the Hurst number of best fit using Eq. (10).

Because the series considered in the preceding paragraphs is binary, it is possible to interpret the scaling constants $a_{i}$ $(I=1,2)$ as follows (cf. Jenkins and Watts, 1968; Agterberg, 1974). Suppose the two binary states along the borehole are written as +1 and -1 . If the mean can be set equal to zero, the autocorrelation $\rho(h)$ is equal to the sum of the probability 


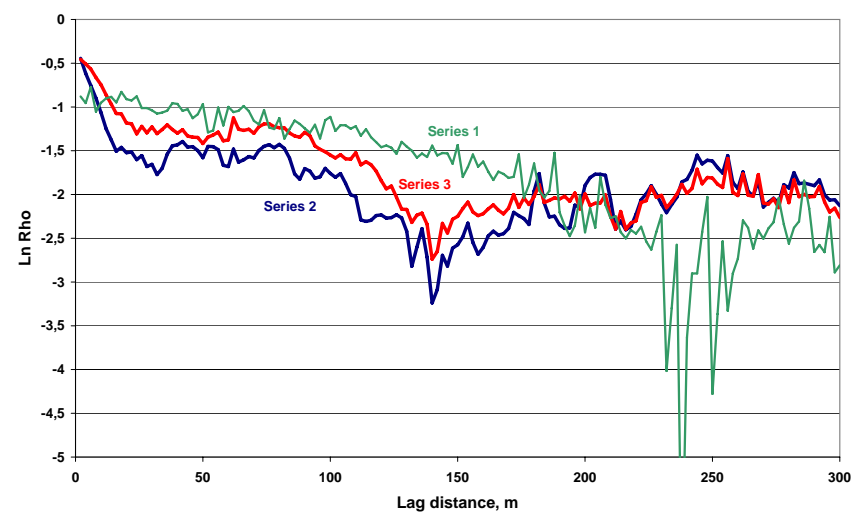

Fig. 16. Correlograms for three consecutive series of copper concentration (ppm) values from Main KTB borehole. Series 2 (for depths between 2 and $4 \mathrm{~km}$ ) and Series 3 (for depths between 4 and $5.54 \mathrm{~km}$ ) show similar autocorrelation functions that differ from autocorrelation function for Series 1 (for depths between $0.05 \mathrm{~km}$ and $2 \mathrm{~km})$.

that number of state changes over the interval $h$ is even minus the sum of the probability that it is odd. If $P_{k}$ represents the Poisson-type probability that there exist $k$ state changes over $h$ :

$\rho(h)=\sum_{k=0}^{\infty}\left(P_{2 k}-P_{2 k-1}\right) ; P_{k}=\frac{e^{-\lambda h}(\lambda h)^{k}}{k !}$

After some manipulation, it follows that

$\rho(h)=e^{-2 \lambda h}$

where $\lambda$ is number of state changes per unit of distance. A similar result is obtained when the mean is not equal to zero.

For the Goff-Hollinger KTB example, the fact that there are two separate negative exponentials illustrates that, over short distances, there are rapid lithology changes or a "nugget effect" for $i=2$, but changes at larger scale are controlled by the other negative exponential $(i=1)$ function. Thus alternation between mostly metabasite and mostly felsic gneisses in KTB is subject to two separate random processes. The alternation either has high or low frequency with probabilities controlled by the $c_{i}(i=1,2)$ coefficients. This type of modeling only applies to the binary residual $V_{p}$ profile for KTB. For example, Marsan and Bean (1999, 2003) have demonstrated that the KTB sonic log can be modeled using a multifractal approach. Also, Hollinger and Goff (2003) have developed a generic model for the $1 / f$ nature of seismic velocity fluctuations. In that paper, these authors modeled the autocovariance function of KTB depthdetrended sonic log through the superposition of four von Kármán autocovariances using negative exponentials with Hurst numbers $v=0.5$ for large, medium, and intermediate scales but $v=0.99$ for the small scale.

\subsection{Detection of nugget effect in copper determinations from the KTB borehole}

The second example is for a long series consisting of 2796 copper (XRF) concentration values for cutting samples taken at $2 \mathrm{~m}$ intervals along the Main KTB borehole already discussed in the previous section. These data are in the public domain (citation: KTB, WG Geochemistry). Depths of first and last cuttings used for this series are $8 \mathrm{~m}$ and $5596 \mathrm{~m}$, respectively. Locally, in the database, results are reported for a 1-m sampling interval; then, alternate copper values at the standard $2 \mathrm{~m}$ interval were included in the series used for example. Most values are shown in Fig. 15 together with a 101-point average representing consecutive 202-m long segments of drill-core. The data set was divided into three series (1, 2 and 3) with 1000, 1000 and 796 values, respectively. Mean copper values for these three series are 37.8, 33.7 and $39.9 \mathrm{ppm} \mathrm{Cu}$, and corresponding standard deviations are 20.3, 11.0 and $20.6 \mathrm{ppm} \mathrm{Cu}$, respectively. Fig. 16 shows correlograms of the three series. Each series shows a nugget effect that, for series 2 and 3, is accompanied by a relatively steeply increasing curve near the origin. Because the autocorrelation coefficients are logarithmically transformed, random fluctuations for near-zero autocorrelation values are amplified. It is noted, however, that all three series only had positive autocorrelations for the first 150 lag distances. Also, the patterns for series 2 and 3 are strikingly similar.

It can be expected that series of element concentrations over a vertical distance of about $5.5 \mathrm{~km}$ will exhibit deterministic trends reflecting systematic changes in rock compositions. It is assumed here that these trends are largely captured by the moving average curve of Fig. 15. Figure 17 shows autocorrelation coefficients for the three series after subtracting the trend values from the original data. All three series of deviations have autocorrelation functions that are approximately negative exponential in shape over distances less than $10 \mathrm{~m}$. Each can be regarded as representing a nugget effect with equation $\rho_{h}=c \exp (-a h)$. The slope coefficients (a) of the three curves are nearly equal to one another $(0.40$, 0.38 and 0.41 for series 1,2 and 3 , respectively). The spatial extent of this nugget effect is much less than the small scale binary lithology variation for the same borehole discussed in Sect. 4.3. It is interesting that the parameter $(a)$ that determines the spatial extent of the nugget effect remains the same over a vertical distance of nearly $6 \mathrm{~km}$. The corresponding variance components $(c)$ of the copper nugget effect are 0.46 , 0.82 and 0.81 , indicating that the white noise component is relatively strong for series 1 .

Quantitative modeling of the nugget effect in KTB copper determinations has yielded better results than could be obtained for our examples from mineral deposits including the Pulacayo Mine. This is not only because the series of chemical determinations is much longer but also because the nugget effect remains clearly visible over lag distances between $2 \mathrm{~m}$ (= original sampling interval) and $10 \mathrm{~m}$. 


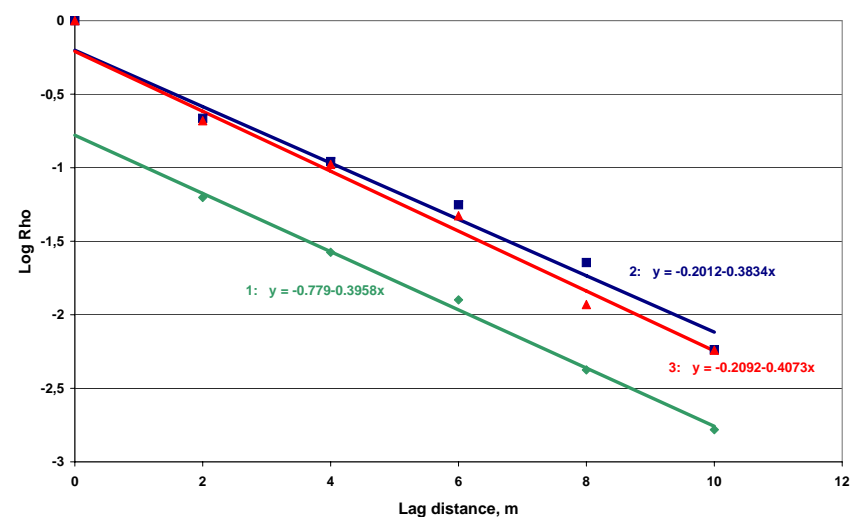

Fig. 17. Correlograms (first 5 lag distances only) for three series of differences between original copper concentration values and mean values shown in Fig. 15. Results are for same series as used for Fig. 16 (Series 1: green; Series 2: black; Series 3: red). Best-fitting semi-exponentials were obtained by ordinary least squares method (Logarithms base 10). The slopes of the three best-fitting straight lines are nearly equal. This indicates existence of a nugget effect with same spatial extent along the entire Main KTB borehole.

\section{Local singularity analysis}

\subsection{Basic concepts of singularity analysis}

Cheng $(1999,2005)$ has proposed a new model for incorporating spatial association and singularity in interpolation of exploratory data. In his approach geochemical or other data collected at sampling points within a study area are subjected to two treatments. The first of these is to construct a contour map by any of the methods such as kriging or inverse distance weighting techniques generally used for this purpose. Secondly, the same data are subjected to local singularity mapping. The local singularity $\alpha$ then is used to enhance the contour map by multiplication of the contour value by the factor $\varepsilon^{\alpha-2}$ where $\varepsilon<1$ represents a length measure. A note on notation is in order at this point. In this paper, Matheron's absolute dispersion is written as italic $\alpha$, singularity as normal $\alpha$ and, later in this paper, bold italic $\alpha$ will denote Lévy index. In Cheng's (2005) approach to predictive mapping, the factor $\varepsilon^{\alpha-2}$ is greater than 1 in places where there has been local element enrichment or by a factor less than 1 where there has been local depletion. Local singularity mapping can be useful for the detection of geochemical anomalies characterized by local enrichment even if contour maps for representing average variability are not constructed (cf. Cheng and Agterberg, 2009; Zuo et al., 2009).

According to Chen et al. (2007) local scaling behaviour follows the following power-law relationship:

$\rho\left\{B_{x}(\varepsilon)\right\}=\frac{\mu\left\{B_{x}(\varepsilon)\right\}}{s^{E}}=c(x) \varepsilon^{\alpha(x)-E}$

where $\rho\left\{B_{x}(\varepsilon)\right\}$ represents element concentration value determined on a neighbourhood size measure $B_{x}$ at point $x$,
$\mu\left\{B_{x}(\varepsilon)\right\}$ represents amount of metal, and $E$ is the Euclidean dimension of the sampling space. For our 1-dimensional $\mathrm{Pu}-$ lacayo example, $E=1$; and, for $\varepsilon=1, B_{x}$ extends $\varepsilon / 2=1 \mathrm{~m}$ in two directions from each of the 118 points along the line parallel to the mining drift. Suppose that average concentration values $\rho\left\{B_{x}(\varepsilon)\right\}$ are also obtained for $\varepsilon=3,5,7$ and 9 , by enlarging $B_{x}$ on both sides. The yardsticks $\varepsilon$ can be normalized by dividing the average concentration values by their largest length (=9). Reflection of the series of 118 points around its first and last points can be performed to acquire approximate average values of $\rho\left\{B_{x}(\varepsilon)\right\}$ at the first and last 4 points of the series. Provided that the model of Eq. (13) is valid, a straight line fitted by least squares to the 5 values of $\ln \mu\left\{B_{x}(\varepsilon)\right\}$ against $\alpha(x) \ln \varepsilon$ then provides estimates of both $\ln c(x)$ and $\alpha(x)$ at each of the 118 points. Estimates of $c(x)$ and $\alpha(x)$ are shown in Fig. 18 (red line) and Fig. 20 (Series $1)$, respectively. These results of ordinary local singularity mapping duplicate estimates previously obtained by Chen et al. (2007) who proposed an iterative algorithm to obtain improved estimates. Their rationale for this was as follows.

In general, $\rho\left\{B_{x}(\varepsilon)\right\}$ is an average value of element concentration values for smaller $B$ 's at points near $x$ with different local singularities. Consequently, use of Eq. (13) would produce biased estimates of $c(x)$ and $\alpha(x)$. How could we obtain estimates of $c(x)$ that are non-singular in that they are not affected by the differences between local singularities within $B_{x}$ ? Chen et al. (2007) proposed to replace Eq. (13) by:

$\rho(x)=c^{*}(x) \varepsilon^{\alpha^{*}(x)-E}$

where $\alpha^{*}(x)$ and $c^{*}(x)$ are the optimum singularity index and local coefficient, respectively. The initial crude estimate $c(x)$ obtained by Eq. (13) at step $k=1$ is refined repeatedly by using the iterative procedure:

$c_{k-1}(x)=c_{k}(x) \epsilon^{\alpha_{k}(x)-E}$

Equation (15) is similar to Eq. (3) of Chen et al. (2007). Employing the previous least squares fitting procedure at each step resulted in the values of $c_{k}(x)$ shown in Fig. 18 for the first and fourth step of the iterative process, and for $k=1000$ after convergence has been reached. Our values for the first four steps of the iterative process exactly duplicated Chen et al. (2007)'s values plotted in their Fig. 1 and partially listed in their Table 1 except for the first and last 4 values in all successive series because a slightly different end correction was employed (see before). For $k=1$, the pattern of $c_{k}(x)$ resembles the signal in our Fig. 1 that was obtained previously by eliminating the noise component from the 118 zinc values. Chen et al. (2007) selected $\alpha^{*}(x)=\alpha_{4}$ $(x)$ because at this point the rate of convergence has slowed down considerably.

\subsection{Extension of local singularity iteration algorithm}

For this paper, the iterative process was continued until approximately full convergence was reached in order to obtain 


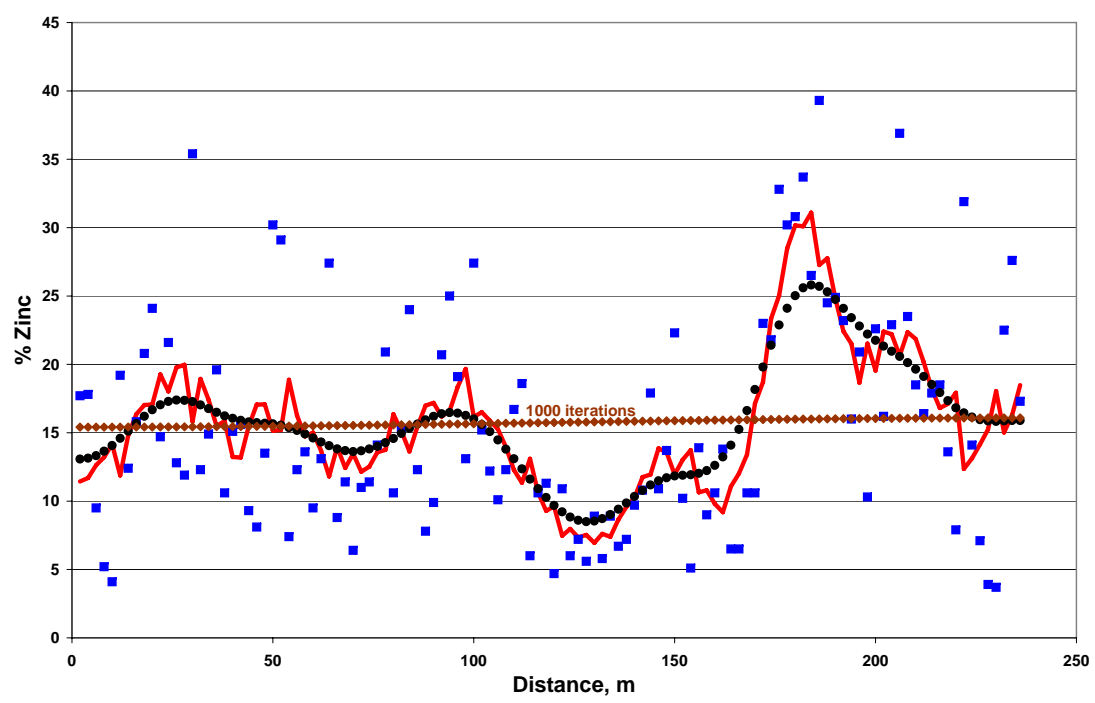

Fig. 18. Results of applying iterative method of Chen et al. (2007) augmented by continuing iteration process until full convergence is reached. Original zinc values (blue) are being smoothed during successive iterations. Second series (red line) obtained after first iteration resembles "signal" in Fig. 1. Values obtained after 4 iterations are shown as black diamonds. At the end of the process, after 1000 iterations when convergence has been reached, the result is approximately a straight line (brown diamonds) with average value slightly below average zinc content $(=15.61 \% \mathrm{Zn})$.

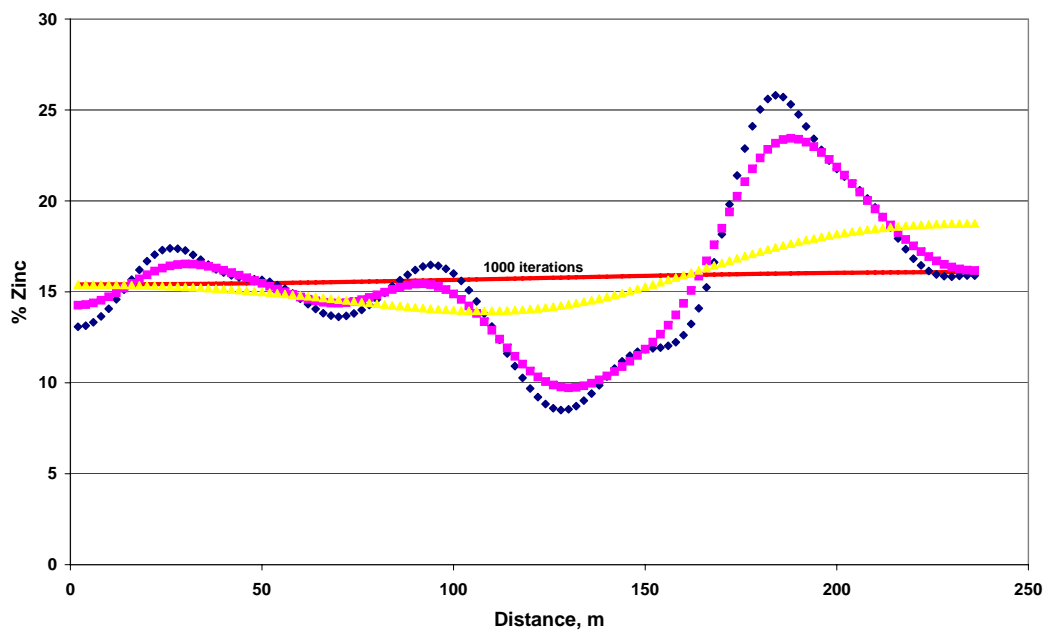

Fig. 19. Same as Fig. 18 but iterative process was constrained to preserve average zinc value of $15.61 \%$. Result after 4 iterations $(k=5)$ is same as in Fig. 18 but result after 1000 iterations $(k=1000)$ is slightly different. Intermediate steps for $k=10$ and 100 are also shown.

more complete information on the nugget effect. In the limit, after about 1000 iterations, the final pattern is as shown in Fig. 18 with an average value that is slightly less than $15.61 \% \mathrm{Zn}$ representing the average of the 118 input values. This bias is due to the fact that, at each step of the iterative process, straight-line fitting is being applied to logarithmically transformed variables and results are converted back to original data scale. The small bias can be avoided by forcing the mean to remain equal to $15.61 \%$ during all steps of the iterative process. End product and some intermediate steps of this new run are shown in Fig. 19. In comparison with Fig. 18, the output for series obtained after the first and fourth step of the iterative process remains unchanged. There is a very small difference in results for $k=1000$. This confirms that in local singularity analysis it is generally permitted to neglect bias introduced by logarithmic transformation of variables. In Sect. 3 it was mentioned that the variance of values used in least squares straight line fitting ranges from $\sigma_{\mathrm{E}}^{2}=0.0622$ to $\sigma_{\mathrm{E}}^{2} / 9=0.0069$ at the beginning of the iterative process, and these variances remain very small at later steps. Estimated singularities for $k=1$, 4 and 1000 are shown in Fig. 20. The results for $k=1$ and 


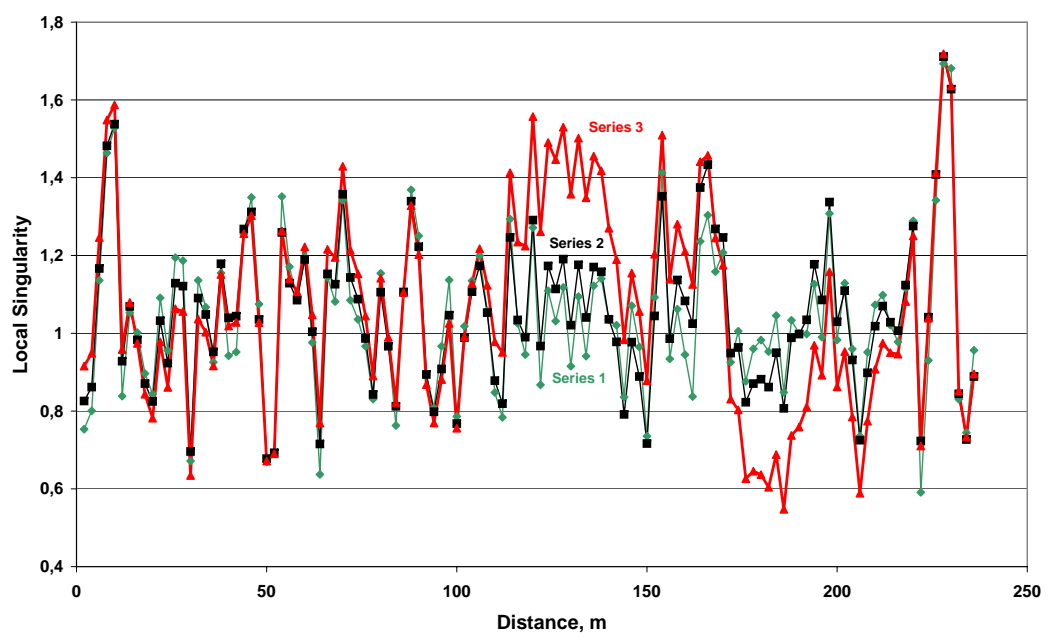

Fig. 20. Estimated singularities for iterative process with $k=1$ (green diamonds of Series 1 ), $k=5$ (black squares of Series 2 )and $k=1000$ (red triangles of Series 3 ). Final singularities $(k=1000)$ differ only slightly from local singularities estimated by means of Eq. (17) $(k=1)$. Except near beginning and end (locations $1-4$ and 115-118) results are identical to estimates of Chen et al. (2007). For $k=1000$, original iterative process of Fig. 18 and modified process of Fig. 19 gave the same final singularities.

$k=4$ duplicate the results previously obtained by Chen et al. (2007).

Full convergence singularities $(k=1000)$ are significantly different from local singularities and results for $k=4$ differ in two neighbourhoods along the Tajo vein (approximately from sampling point positions 60 to 75 , and 90 to 100 , respectively). In Fig. 21, final singularities are plotted against original zinc concentration values showing a logarithmic curve pattern. In Fig. 22 a straight line of least squares was fitted for final singularity versus $\log _{10}(\% \mathrm{Zn})$ with the residuals (deviations from this best fitting line) shown separately in Fig. 23. The residuals exhibit a white noise pattern with variance equal to 0.001178 . Using original zinc values, the variance of residuals is estimated to be 1.3837 . Because \% $\mathrm{Zn}$ variance is 64.13 , it follows that the white noise component is 0.02079 . This is only about 4 per cent of the variance of the noise component previously used to construct the signal of Fig. 1. The new sampling error could be a measurement error of the original chemical determinations for zinc and incorporate the crystal boundary effect (Sect. 4.1). For our example, incorporation of the nugget effect to estimate zinc content (e.g. by using the theoretical values on the curve fitted in Fig. 21), approximately reproduces the observed values. This in itself is a trivial result. However, the example illustrates that, in general, any of the moving average techniques, e.g. those commonly used to construct contour maps from measurements at points outside the sphere of influence of the nugget effect (cf. Fig. 12), can be improved by incorporating information on local singularities as originally proposed by Cheng (2005).

Local singularity is associated with variability over very short distances or "nugget effect". Singularities less than 1 signify local $\mathrm{Zn}$ enrichment whereas singularities greater

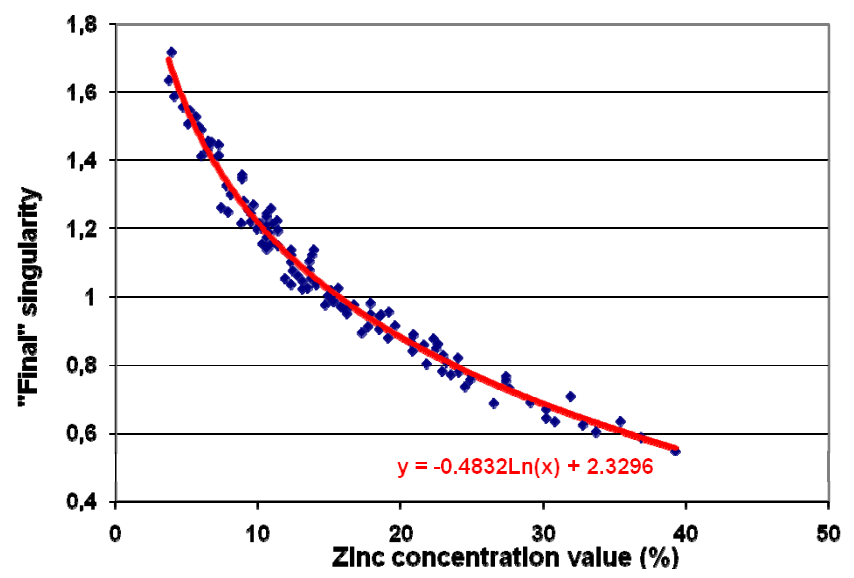

Fig. 21. Relationship between final singularity and zinc concentration value is logarithmic. Final singularities (blue diamonds) are same as those for Series 3 in Fig. 20. Logarithmic curve (red line) was fitted by least squares.

than 1 indicate depletion. Minimum and maximum singularities are $\alpha_{\min }=0.547$ and $\alpha_{\max }=1.719$, respectively. These values are only slightly different from $\alpha_{\min }=0.591$ and $\alpha_{\max }=1.693$ obtained using Eq. (15) (also see Chen et al., 2007, Table 1). They differ more strongly from $\alpha_{\min }=0.835$ and $\alpha_{\max }=1.402$ derived by Cheng and Agterberg (1996) on the basis of the binomial $/ p$ model fitted to the 118 zinc values. The newly derived values $\left(\alpha_{\min }=0.547\right.$ and $\left.\alpha_{\max }=1.719\right)$ are probably better indicating that the original binomial/ $p$ multifractal spectrum (Cheng and Agterberg, 1996, Fig. 2d) is probably too narrow. 


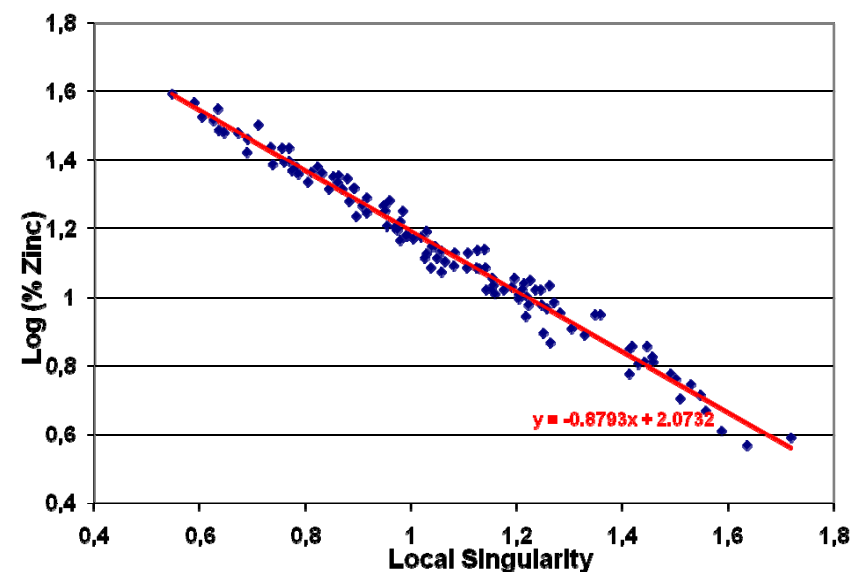

Fig. 22. Final singularity (black diamonds) plotted against $\log _{10}(\%$ $\mathrm{Zn}$ ) is according to straight line (red) fitted by least squares.

\section{Multifractal modeling and spectral analysis}

\subsection{Binomial $/ p$ model}

Theory of the binomial/ $p$ model is presented in textbooks including Feder (1988), Evertsz and Mandelbrot (1992), Mandelbrot (1999) and Falconer (2003). There have been numerous successful applications of this relatively simple model including many to solve solid Earth problems (e.g. Cheng, 1994; Cheng and Agterberg, 1996; Agterberg, 2007; Xie et al., 2007; Cheng, 2008). Although various departures from the model have been described in these papers and elsewhere, the binomial $/ p$ model basically is characterized by a single parameter. In the original model of de Wijs (1951), this parameter is the dispersion index $d$. In the Introduction it was discussed that the absolute dispersion of Matheron satisfies $\alpha=(\ln \eta)^{2} / \ln 16$, and $\eta=(1+d) /(1-d)$. When the parameter $p$ is used, we have $p=0.5(1-d)$. The multifractal spectrum of this model has its maximum $f(\alpha)=1$ (for $E=1$ ) at $\alpha=1$, and $f(\alpha)=0$ at:

$\alpha_{\min }=\log _{2}(1-p) ; \alpha_{\max }=-\log _{2} p$

Another parameter that can be used to characterize the binomial $/ p$ model is the second order mass exponent $\tau(2)=$ $-\log _{2}\left\{p^{2}+(1-p)^{2}\right\}$. If the binomial $/ p$ model is satisfied, anyone of the parameters $p, d, \alpha, \tau(2), \alpha_{\min }, \alpha_{\max }$, or $\sigma^{2}$ (ln $x$ ) can be used for characterization. Using different parameters is helpful in finding significant departures from model validity.

In the preceding section it was noted that estimates of $\alpha_{\min }$ and $\alpha_{\max }$ derived for the Pulacayo orebody in Chen et al. (2007) and in this paper differ greatly from previous estimates based on the binomial $/ p$ model. However, Fig. 3 in Cheng and Agterberg (1996) illustrates that this model provides excellent fit with $95 \%$ confidence interval equal to $\tau(2)=0.979 \pm 0.038$. From this result it would follow

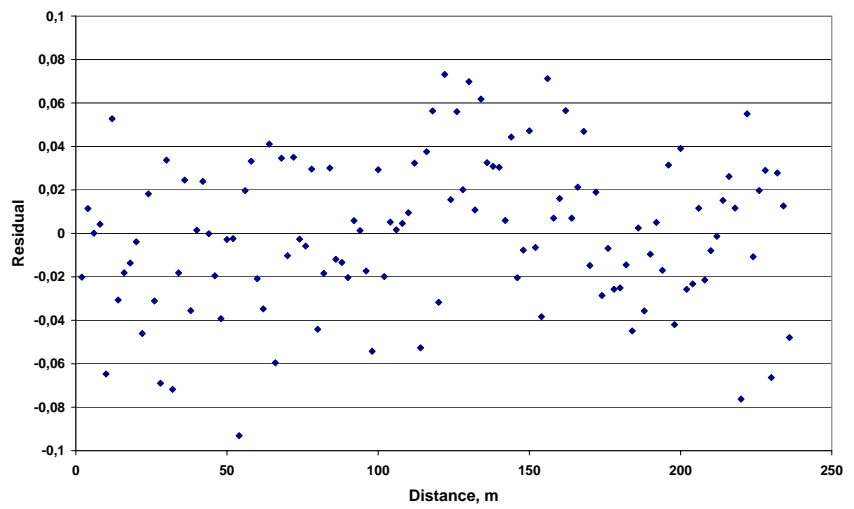

Fig. 23. Residuals from straight line of Fig. 22 show white noise pattern with variance equal to 0.00118 .

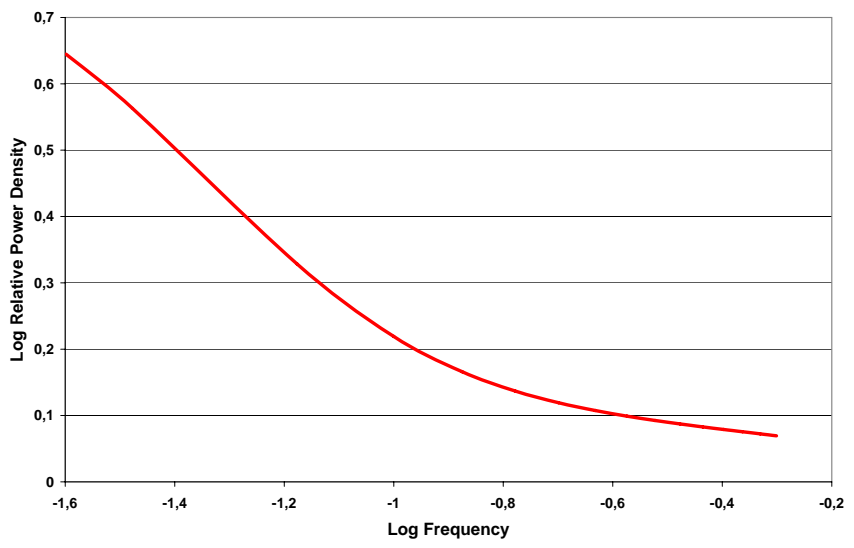

Fig. 24. Relative power spectrum for autocorrelation function shown in Fig. 12. Decrease in slope at higher frequency side is caused by the nugget effect (Logarithms base 10).

that $d=0.121, \alpha_{\min }=0.835$, and $\alpha_{\max }=1.186$. The latter estimate differs not only from $\alpha_{\max }=1.719$ derived in this paper, it also is less than the estimate $\alpha_{\max }=1.402$ on the right side of the multifractal spectrum in Cheng and Agterberg (1996). The estimate $d=0.121$ is much too small. Using absolute values of differences between successive values, de Wijs (1951) had already derived $d=0.205$ resulting in Matheron's $\alpha=0.0208$, and this is close to $\alpha=0.0195$ derived on the basis of Fig. 10 in this paper. Use of any of the estimates of $\alpha_{\min }$ or $\alpha_{\max }$ obtained in the preceding section would result in estimates of $d$ that are much too large; e.g. the full convergence local singularities would yield $d=0.369$ and $d=0.392$, respectively. Clearly, the binomial $/ p$ model has limited range of applicability although it shows linear patterns for different moments $(q)$ on the log-log plot of partition function versus $\epsilon$ when the multifractal spectrum is derived. The preceding inconsistencies suggest that a more flexible model with additional parameters should be used. The "universal multifractal model" with three parameters was initially developed during the late 1990s by Schertzer 
and Lovejoy (1991). Lovejoy and Schertzer (2007) have successfully applied this model to the 118 Pulacayo zinc values as will be discussed in the next section.

\subsection{Universal multifractal modeling}

Figure 3a in Lovejoy and Schertzer (2007) shows a realistic universal multifractal simulation for the Pulacayo orebody using the following three parameters: Lévy index $\boldsymbol{\alpha}=1.8$, codimension $C_{1}=0.03$ and deviation from conservation $H=0.090$. Their approach is explained in detail and illustrated by means of other applications in a large number of publications including Lovejoy, Gaonac'h, and Schertzer (2008), Lovejoy et al. (2001), Schertzer and Lovejoy (editors, 1991), Schertzer and Lovejoy (1996) and Schertzer et al. (1997). The codimension $C_{1}$, which characterizes sparseness of mean field, and $H$ can be derived as follows. First a log-log plot of the so-called "first order structure function" (cf. Monin and Yaglom, 1975) is constructed. Successive moments are obtained for absolute values of differences between concentration values for points that are distance $h$ apart by raising them to the powers $q(=0.25,0.5, \ldots, 3$ for the 118 zinc values). The resulting pattern for $q=2$ represents the variogram and the first point on the pattern for $q=0$ is the de Wijs index of dispersion $d$. Straight lines are fitted to all patterns and a new diagram is constructed with the slopes of the lines $\left(\xi_{q}\right)$ plotted against $q$. Slope and value of this new line near $q=1$ yielded $H=0.090$ and $C_{1}=0.02$ for the Pulacayo orebody because $H=\xi_{1}$ and $C_{1}=H-\xi_{1}^{\prime}$ where $\xi_{1}^{\prime}$ is the first derivative of $\xi_{q}$ with respect to $q$ (Lovejoy and Schertzer, 2007, Fig. 26a and b). Their use of the so-called "double trace moment" method (cf. Lavallée et al., 1992) yielded estimates of the Lévy index equal to $\boldsymbol{\alpha}=1.76$ and $\boldsymbol{\alpha}=1.78$, and codimension $C_{1}=0.023,0.022$, respectively. In general, a relatively small value of $C_{1}$ with respect to $H$ indicates that the multifractality is so weak that deviation from conservation $(H)$ will be dominant except for quite high moments (Lovejoy and Schertzer, 2007, p. 491). In the preceding section it was shown that the binomial $/ p$ model produced inconsistencies between results for lower and higher moments. Universal multifractal modeling is more flexible and produces realistic zinc concentration variability. On the other hand, the estimate for the second order moment $(\tau(2)=0.979 \pm 0.038)$ produces a realistic autocorrelation function including the nugget effect, which affects the power spectrum for high frequencies as will be discussed in the next section. The existence of $\tau(2)$ as a constant parameter is not tied to validity of the binomial $/ p$ model for both lower and higher moments (Sect. 4.2).

\subsection{Spectral analysis}

Another important tool in universal multifractal modeling is spectral analysis. Theoretically, this model results in a

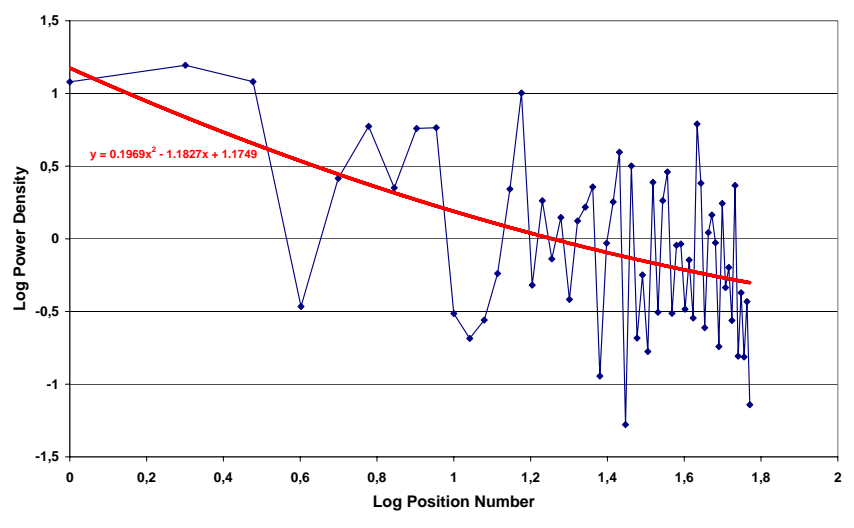

Fig. 25. Periodogram (black diamonds) of 118 zinc values with quadratic curve (red curve) fitted by least squares (Logarithms base 10). The flattening of the curve toward higher frequencies is due to the nugget effect (Logarithms base 10).

spectrum consisting of a straight line with slope $-\boldsymbol{\beta}$. This parameter can either be estimated directly or indirectly using $\boldsymbol{\beta}=1-K_{2}+2 H$ where $K_{2}$ representing the "second characteristic function". Lovejoy and Schertzer (2007) estimated $K_{2}=0.05$ by double trace moment analysis. With the previously mentioned estimate $H=0.090$ this yielded $\boldsymbol{\beta} \approx 1.12$ in good agreement with the experimental spectrum for the 118 zinc values.

Spectral analysis of the 118 logarithmically zinc values was performed previously (Agterberg, 1967, Fig. 4). The discrete Fourier transform was taken of autocorrelation correlation coefficients with lag distances $<32 \mathrm{~m}$ after applying a cosine transformation in order to largely eliminate distortions according to Tukey's "hanning" method (Blackman and Tukey, 1958). In a discussion of this result, Tukey (1970) pointed out that the resulting spectrum "drooped" although it was within the $90 \%$ confidence interval around the theoretical spectrum for the signal-plus-noise model with negative exponential autocorrelation function (cf. Fig. 5). Replotting the earlier results on a log-log plot shows a linear pattern with straight line of best fit yielding $\boldsymbol{\beta}=0.79$.

The normalized power spectrum corresponding to Eq. (6) is:

$P(f)=\Sigma_{i=1}^{2}\left[1-c_{i}+\frac{c_{i} / \pi f_{c i}}{1+\left(f / f_{c i}\right)^{2}}\right]$

where $f_{c i}=a_{i} / 2 \pi$. A log-log plot of this spectrum is shown in Fig. 24 adopting the coefficients previously used for the autocorrelation function satisfying Eq. (6) and plotted in Fig. 12. The curve in Fig. 24 is approximately a straight line for lower frequencies but for high frequencies there is a marked decrease of slope reflecting the nugget effect.

Figure 25 shows the periodogram of the 118 zinc values together with a quadratic curve fitted by least squares. A bestfitting straight line for the same values results in $\beta=0.72$, but by means of an F-test it can be shown that the quadratic 


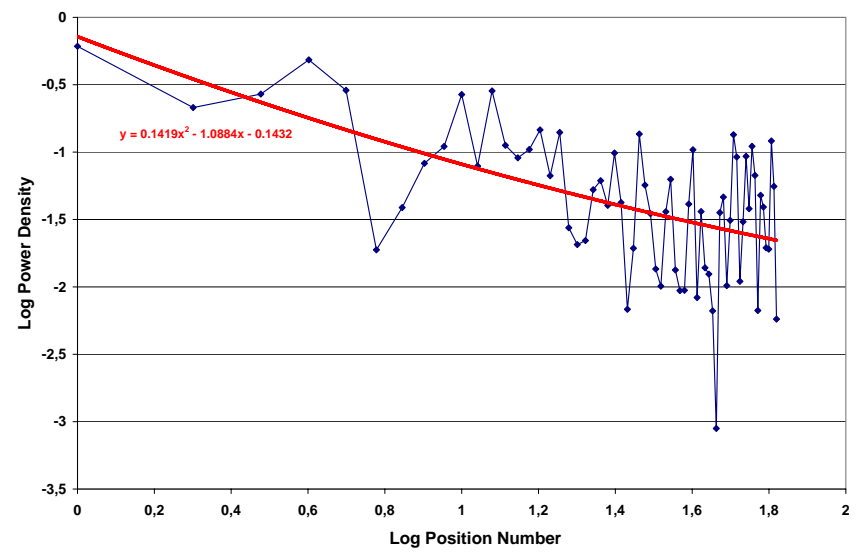

Fig. 26. Periodogram (black diamonds) of $132 \mathrm{TiO}_{2}$ concentration values from boreholes in the Black Cargo Titaniferous magnetite deposit, Los Angeles County, California. Quadratic curve (red curve)was fitted by least squares. The overall pattern is similar to that of Fig. 25 for the Pulacayo zinc values.

fit is significantly better than the linear fit in Fig. 25 (for level of significance $=0.01$ ). The slope of the curve at the origin in Fig. 25 gives $\beta=1.18$ with gradually decrease to 0.49 at maximum log wave number on the right. A log-log plot of the 2-point moving average of the periodogram of Fig. 21 produces a pattern that is close to Lovejoy and Schertzer's (2007, Fig. 3b) spectrum for the de Wijs data. A straight line fit to the first 20 points of this 2-point moving average gives $\boldsymbol{\beta}=1.03$, which is close to $\boldsymbol{\beta}=1.18$ at the origin of Fig. 25 and close to $\beta \approx 1.12$. A possible explanation is that spectral analysis confirms validity of the universal multifractal model but with superimposed noise that tends to flatten the spectrum at higher frequencies. For comparison, the preceding method also was applied to a sequence of 132 titanium concentration values from the Black Cargo Titaniferous magnetite deposit, Los Angeles County, California (Benson et al., 1962). This sequence, previously analyzed in Agterberg (1965), is a composite of four sub-sequences obtained from 4 different boreholes. All samples were $5 \mathrm{ft}$ in length except for three $10 \mathrm{ft}$ samples at the subsequence meeting points. Mean and standard deviation of the 132 numbers are $2.73 \%$ and $1.65 \% \mathrm{TiO}_{2}$, respectively. The resulting periodogram shown in Fig. 26 is similar to Fig. 25 in that the best-fitting quadratic trend line has a slope that decreases toward higher frequencies. At the origin $(x=0)$ its value is -1.088 and at maximum frequency $(x=1.8195)$ the slope is -0.6186 . Other results for this example also were similar to those obtained for the 118 Pulacayo zinc values.

The curves in Figs. 23 to 25 indicate (1) the log-log plots of the three power spectra are not straight lines but curves with slopes that decrease toward higher frequencies; and (2) at their maximum frequency or highest position number the curves are probably not horizontal indicating that the nugget effect is not white noise with Dirac delta autocorrelation function. The sampling intervals of two data sets used for example in this section are too wide to allow a better description of the effect of the nugget effect on the power spectra.

\section{Concluding remarks}

In a general way, orebodies are different from most other rocks (sedimentary, igneous, volcanic, metamorphic rocks) in that most of them exhibit clear evidence that nonlinear processes were involved in their genesis. This evidence includes the following features: (1) element concentration values in orebodies commonly show a positively skewed frequency distribution that is approximately lognormal; (2) element concentration values in orebodies generally exhibit spatial covariance including a nugget effect at or near the origin; (2) statistics that apply to entire orebodies of the same type including total amount of ore usually show approximately lognormal distributions in the vicinity of their means and medians but the high-value tails of these frequency distributions can be Pareto-type; and (3) bedrock and surficial geology in the vicinity of orebodies often show patterns with characteristics similar to those of concentration values within the mineral deposits. All of these four features are of economic importance: (1) and (2) are important for ore reserve estimation; (2) facilitates regional or global mineral potential estimation; and (3) is helpful in prospecting for undiscovered deposits. In a general way, these rules apply to hydrocarbon deposits as well (cf. Barton and La Pointe, editors).

Although the preceding four features have been known to exist for a long time, it is only relatively recently that nonlinear methods to clarify the spatial element concentration patterns and their genesis have become available. The primary purpose of this paper was to help build a bridge between Matheron-type geostatistical sampling methodologies and geometric probability theory with nonlinear process modeling methods. Main conclusions are as follows:

1. Existing sampling techniques applied within known orebodies such as channel sampling along mining drifts yield average concentration values for blocks of ore that have relatively small extension variance and can be used for multifractal modeling in addition to their usage in existing ore reserve estimation approaches.

2. Geometric probability theory applied to Matheron's extension of the model of de Wijs suggests that new information on the nugget effect consisting of local clustering of ore minerals can be derived from experimental variograms.

3. The new method of local singularity mapping applied within orebodies provides new insights into the nature of the nugget effect which has spatial extent less than distances between samples collected for chemical analysis; within the Pulacayo orebody, Bolivia, local 
singularity is linearly related with logarithmically transformed concentration value.

4. Parameters to characterize spatial covariance, which are estimated from samples subjected to chemical analysis, are not necessarily valid at strictly local scale because of the nugget effect; this indicates that moving averages resulting from covariance models or estimated by other methods such as kriging then can be improved by incorporating local singularities for additional local element enrichment or depletion to be superimposed on the smooth moving average patterns.

5. Although the series of 118 zinc concentration values from the Pulacayo Mine was the primary example in this paper, similar negative exponential autocorrelation functions were found for copper concentration values from channel samples from the Whalesback copper Mine, Newfoundland, and gold in Witwatersrand Mines, South Africa although the nugget effect could not be studied in more detail for these examples. In examples of application to large KTB borehole data sets, nugget effects were shown to exist in binary residual $V_{p}$ profile for lithology (alternating mostly gneiss and metabasite layers) over a length of approximately $7 \mathrm{~km}$, and in copper concentration values over nearly $6 \mathrm{~km}$.

6. Although there have been many successful applications of the multifractal binomial/ $p$ model, its application within the Pulacayo orebody results in several inconsistencies indicating shortcomings of this relatively simple approach.

7. Universal multifractal modeling is a promising new approach to improve upon use of the binomial/ $p$ model. However, more research will be needed to clarify how spectral analysis and universal multifractal modeling can be applied to orebodies and their surroundings.

Acknowledgements. Thanks are due to Zhijun Chen, Qiuming Cheng, John Goff, Shaun Lovejoy, Stephen Morris and an anonymous reviewer for helpful comments and discussion. Help from Kim Nguyen in preparation of the figures is gratefully acknowledged. The Central Division, Geological Survey of Canada has provided financial support for publication costs.

Edited by: U. Feudel

Reviewed by: J. Goff and another anonymous referee

\section{References}

Agterberg, F. P.: The skew frequency-curve of some ore minerals, Geologie en Mijnbouw, 40, 149-162, 1961.

Agterberg, F. P.: The technique of serial correlation applied to continuous series of element concentration values in homogeneous rocks, J. Geol., 73, 142-154, 1965.
Agterberg, F. P.: Mathematical models in ore evaluation, J. Can. Oper. Res. Soc., 5, 44-158, 1967.

Agterberg, F. P.: Geomathematics, Elsevier, Amsterdam, 596 pp., 1974.

Agterberg, F. P.: Fractals, multifractals and change of support, in: Geostatistics for the Next Century, edited by: Dimitrakopoulos, R., Kluwer, Dordrecht, 223-234, 1994.

Agterberg, F. P.: New applications of the model of de Wijs in regional geochemistry, Math. Geol., 39, 1-25, 2007a.

Agterberg, F. P.: Mixtures of multiplicative cascade models in geochemistry, Nonlin. Processes Geophys., 14, 201-209, doi:10.5194/npg-14-201-2007, 2007b.

Ahlfeld, F.: Los Yacimientos Minerales de Bolivia, Imprenta Industrial, Bilbao, S.A., 277 pp., 1954.

Aitchison, J. and Brown, J. A. C.: The Lognormal Distribution, Cambridge Univ. Press, 176 pp., 1957.

Barnsley, M: Fractals Everywhere, Academic Press, Boston, 394 pp., 1988.

Barton, Ch. C. and La Pointe, P. R. (Eds.): Fractals in Petroleum Geology and Earthy Processes, Plenum, New York, 317 pp., 1995.

Benson, W. T., Engel, A. L., and Heinen, H. J.: Titaniferous magnetite deposits, Los Angeles County, Calif., US Bureau of Mines, Rept. of Investigations 5962, 1962.

Blackman, R. B. and Tukey, J. W.: The Measurement of Power Spectra, 190 pp., Dover, New York, 1958

Chemingui, N.: Modeling 3-D anisotropic fractal media, Stanford Exploration Project, Rept. 80, May 15, 1-13, 2001.

Zhijun Chen, Qiuming Cheng, Jiaoguo Chen, and Shuyun Xie: A novel iterative approach for mapping local singularities from geochemical data, Nonlin. Processes Geophys., 14, 317-324, doi:10.5194/npg-14-317-2007, 2007.

Cheng, Q.: Multifractal interpolation, in: Proc. Fifth Annual Conf. Internat. Ass. Math. Geol., edited by Lippard, S. J., Naess, A., and Sinding-Larsen, R., Trondheim, Norway, 245-250, 1999.

Cheng, Q.: A new model for incorporating spatial association and singularity in interpolation of exploratory data, Geostatistics Banff 2004, edited by: Leuanthong, D. and Deutsch, C. V., Springer, Dordrecht, the Netherlands, 1017-1025, 2005.

Cheng, Q.: GIS based fractal/multifractal anomaly analysis for modeling and prediction of mineralization and mineral deposits, in: GIS for the Earth Sciences, edited by: Harris, J. R., Geol. Ass. Canada, Toronto, Canada, 131-142, 2006.

Cheng, Q.: GIS based fractal and multifractal methods for mineral deposit prediction, in: Non-linear theory and power-law models for information integration and mineral resources quantitative assessments, in: Progress in Geomathematics, edited by: BonhamCarter, G. and Cheng, Q., Springer, Heidelberg, 195-225, 2008.

Cheng, Q. and Agterberg, F. P.: Multifractal modelling and spatial statistics, Math. Geol., 28, 1-16, 1996.

Cheng, Q. and Agterberg, F. P.: Singularity analysis of ore-mineral and toxic trace elements in stream sediments, Comput. Geosci., 35, 234-244, 2009.

Cressie, N. A. C.: Statistics for Spatial Data, Wiley, New York, 900 pp., 2001.

David, M.: Geostatistical Ore Reserve Estimation, Elsevier, Amsterdam, 364 pp., 1977.

De Wijs, H. J.: Statistics of ore distribution, part I, Geologie en Mijnbouw, 13, 365-375, 1951.

Evertsz, C. J. G. and Mandelbrot, B. B.: Multifractal measures, 
Appendix B, in: Chaos and Fractals, edited by: Peitgen, H.-O., Jürgens, H., and Saupe, D., Springer, New York, 921-953, 1992.

Falconer, K.: Fractal Geometry, Wiley, Chichester, 2nd Edn., 337 pp., 2003.

Feder, J.: Fractals, Plenum, New York, 283 pp., 1988.

Goff, J. A. and Hollinger, K.: Nature and origin of upper crustal seismic velocity fluctuations and associated scaling properties: Combined stochastic analyses of KTB velocity and lithology logs, J. Geophys. Res., 104, 13169-13182, 1999.

Goff, J. A. and Hollinger, K.: A generic model for the $1 / f$-nature of seismic velocity fluctuations, edited by: Goff, J. A. and Hollinger, K., Kluwer Academic, New York, 131-154, 2003.

Goff, J. A. and Jennings, J. W.: Improvement of Fourier-based unconditional and conditional simulations for band limited fractal (von Kármán) statistical models, Math. Geol., 31, 627-649, 1999.

Goff, J. A. and Jordan, T. H.: Stochastic modeling of seafloor morphology: Inversion of Sea Beam data for second-order statistics, J. Geophys. Res., 93, 13589-13608, 1988.

Goff, J. A. and Levander, A.: Incorporating "sinuous connectivity" into stochastic models of crustal heterogeneity: Examples from the Lewisian gneiss complex, Scotland, the Franciscan formation, California, and the Hafafit gneiss complex, Egypt, J. Geophys. Res., 1001, 8489-8501, 1996.

Goff, J. A., Hollinger, K., and Levander, A.: Modal fields: A new method for characterization of random seismic velocity heterogeneity, Geophys. Res. Lett., 21, 493-496, 1994.

Jenkins, G. M. and Watts, D. G.: Spectral Analysis and its Applications, Holden-Day, San Francisco, 525 pp., 1968.

Journel, A. G. and Huijbregts, Ch. J.: Mining Geostatistics, Academic Press, New York, 600 pp., 1978.

Klimeš, L.: Correlation functions in random media, Pure Appl. Geophys., 159, 1811-1831, 2002.

Krige, D. G.: Lognormal de-Wijsian Geostatistics for Ore Evaluation, South African Institute Mining Metall, Monograph Series, Geostatistics 1, 1978.

Krige, D. G. and Ueckermann, H. J.: Value contours and improved regression techniques for ore reserve valuations, J. South Afr. Inst. Mining Metall., May 1963, 429-452, 1963.

Krige, D. G., Watson, M. I., Oberholzer, W. J., and du Toit, S. R.: The use of contour surfaces as predictive models for ore values, Proc. Symp. Comput. Appl. Oper. Res. Mineral Industry, 8th, Am. Instit. Mining Metall., Salt Lake City, 127-161, 1969.

Lavallée, D., Lovejoy, S., Schertzer, D., and Schmitt, F.: On the determination of universal multifractal parameters in turbulence, in Topological Aspects of the Dynamics of Fluids and Plasmas, edited by: Moffatt, H. K., Zaslavsky, G. M., Conte, P., and Tabor, M., Kluwer, Dordrecht, 463-478, 1992.

Lovejoy, S. and Schertzer, D.: Multifractals, universality classes and satellite and radar measurements of cloud and rain fields, J. Geophys. Res., 95, 2021-2034, 1990.

Lovejoy, S. and Schertzer, D.: Scaling and multifractal fields in the solid earth and topography, Nonlin. Processes Geophys., 14, 465-502, doi:10.5194/npg-14-465-2007, 2007.

Lovejoy, S., Gaonac'h, H., and Schertzer, D.: Anisotropic scaling models of rock density and the Earth's surface gravity field, in: Progress in Geomathematics, edited by: Bonham-Carter, G. and Cheng, Q., Springer, Heidelberg, 151-193, 2008.

Lovejoy, S., Currie, W. J. S., Tessier, Y., Claereboudt, M. R., Bour- get, E., Roff, J. C., and Schertzer, D.: Universal multifractals and ocean patchiness: phytoplankton, physical fields and coastal heterogeneity, J. Plankton Res., 23, 117-141, 2001.

Mandelbrot, B. B.: The Fractal Geometry of Nature, Freeman, San Francisco, 468 pp., 1983.

Mandelbrot, B. B.: The statistics of natural resources and the law of Pareto, in: Fractals in petroleum geology and the earth sciences, edited by: Barton, C. C. and La Pointe, P. R., Plenum, New York, 1-12, 1995.

Mandelbrot, B. B.: Multifractals and $1 / f$ Noise, Springer, New York, 442 pp., 1999.

Marsan, D. and Bean, C. J.: Multiscaling nature of sonic velocities and lithology in the upper crystalline crust: Evidence from the KTB main borehole, Geophys. Res. Lett., 26, 275-278, 1999.

Marsan, D. and Bean, C.: Multifractal modeling and analyses of crustal heterogeneity, edited by: Goff, J. A. and Hollinger, K., Kluwer Academic, New York, 207-236, 2003.

Matheron, G.: Traité de Géostatistque Appliquée, Mém. Bur. Rech. Géol. Minières, 14, 333 pp., 1962.

Matheron, G.: The theory of regionalized variables and its applications, Cah. Cent. Morphol. Math., 5, 211 pp., 1971.

Matheron, G.: Effet proportionnel et logormalité ou: Le retours du serpent de mer, Note Géostatistique No. 124, 43 pp., 1974.

Matheron, G.: Estimating and Choosing, an Essay on Probability in Practice, Translated by: Hasofer, A. M., Springer, Heidelberg, 140 pp., 1989.

Monin, A. S. and Yaglom, A. M.: Statistical Fluid Mechanics, M.I.T. Press, Cambridge, Mass., 2, 886 pp., 1975.

Schertzer, D. and Lovejoy, S. (Eds.): Non-Linear Variability in Geophysics, Kluwer, Dordrecht, 318 pp., 1991.

Schertzer, D. and Lovejoy, S.: Universal multifractals do exist! Comments on "A statistical analysis of mesoscale rainfall as a random cascade, J. Appl. Met., 36, 1296-1303, 1997.

Schertzer, D., Lovejoy, S., Schmitt, F., Chigiranskaya, Y., and Marsan, D.: Multifractal cascade dynamics and turbulent intermittency, Fractals, 5, 427-471, 1997.

Sichel, H. S.: The estimation of means and associated confidence limits for small samples from lognormal populations, in: Proc. Symp. Math. Stat. Comput. Appl. Ore Valuation, South Afr. Inst. Mining Metall., Johannesburg, 106-122, 1966.

Stanley, H. E. and Meakin, P.: Multifractal phenomena in physics and chemistry, Nature, 335, 405-409, 1988.

Tukey, J. W.: Some further inputs, in Geostatistics, edited by: Merriam, D. F., Plenum, New York, 163-174, 1970.

Turcotte, D. L.: Fractals and Chaos in Geology and Geophysics, Cambridge University Press, 2nd Edn., 398 pp., 1997.

Turneaure, F. S.: The Bolivian Tin-Silver Province, Economic Geol., 66, 215-225, 1971.

Pinto-Vásquez, J.: Volcanic dome-associated precious and base metal epithermal mineralization at Pulacayo, Bolivia, Economic Geol., 88, 697-700, 1993.

Villalpando, B. and Ueno, H.: El yacimiento argentifero de Pulacayo, Bolivia, Memoria del III Coloquio del Instituto de Geologia Economica, Univ. Mayor de San Andres, La Paz, Bolivia, 203-235, 1987.

von Kármán, T.: Progress in the statistical theory of turbulence, J. Mar. Res., 7, 252-264, 1948.

Vistelius, A. B., Agterberg, F. P., Divi, S. R., and Hogarth, D. D.: A Stochastic model for the crystallization and textural analysis of a 
fine-grained stock near Meech Lake, Gatineau Park, Quebec, 62 pp., Geol. Surv. Canada, Paper 81-21, 1983.

Wang, Z.: GIS-based fractal/multifractal modeling of texture in mylonites and banded sphalerite ores, unpublished $\mathrm{PhD}$ thesis, York University, Toronto, 146 pp., 2008.

Wu, S.-S. and Aki, K.: The fractal nature of homogeneities in the lithosphere evidenced from seismic wave scattering, Pure Appl. Geophys., 123, 805-818, 1985.

Wu, R.-S., Xu, Z., and Li, X.-P.: Heterogeneity spectrum and scale anisotropy in the upper crust revealed by the German Continental Deep-Drilling (KTB) holes, Geophys. Res. Lett., 21, 911-914, 1994.
Xie, S., Cheng, Q., Chen, G., Chen, Z., and Bao, Z.: Application of local singularity in prospecting potential oil/gas Targets, Nonlin. Processes Geophys., 14, 285-292, doi:10.5194/npg-14-2852007, 2007.

Xu, D., Cheng, Q., and Agterberg, F.: Scaling property of ideal granitic sequences, Nonlin. Processes Geophys., 14, 237-246, doi:10.5194/npg-14-237-2007, 2007.

Yaglom, A. M.: An Introduction to the Theory of Stationary Functions, Prentice-Hall, Englewood Cliffs, N.J., 235 pp., 1962.

Zuo, R., Cheng, Q., Agterberg, F. P., and Xia, Q.: Application of singularity mapping technique to identify local anomalies using stream sediment geochemical data, a case study from Gangdese, Tibet, western China, Jour. Geochem. Expl., 101, 225-235, 2009. 